Supporting information for

\title{
Glutathione Peroxidase (GPx)-like Antioxidant Activity of Organoselenium Drug Ebselen: Unexpected Complications with Thiol Exchange Reactions
}

\author{
Bani Kanta Sarma and G. Mugesh*
}

Department of Inorganic \& Physical Chemistry, Indian Institute of Science, Bangalore 560 012, India

Email: mugesh@ipc.iisc.ernet.in

Figure S1. ${ }^{77}$ Se NMR spectrum of pure ebselen (1) in $\mathrm{CDCl}_{3}$.

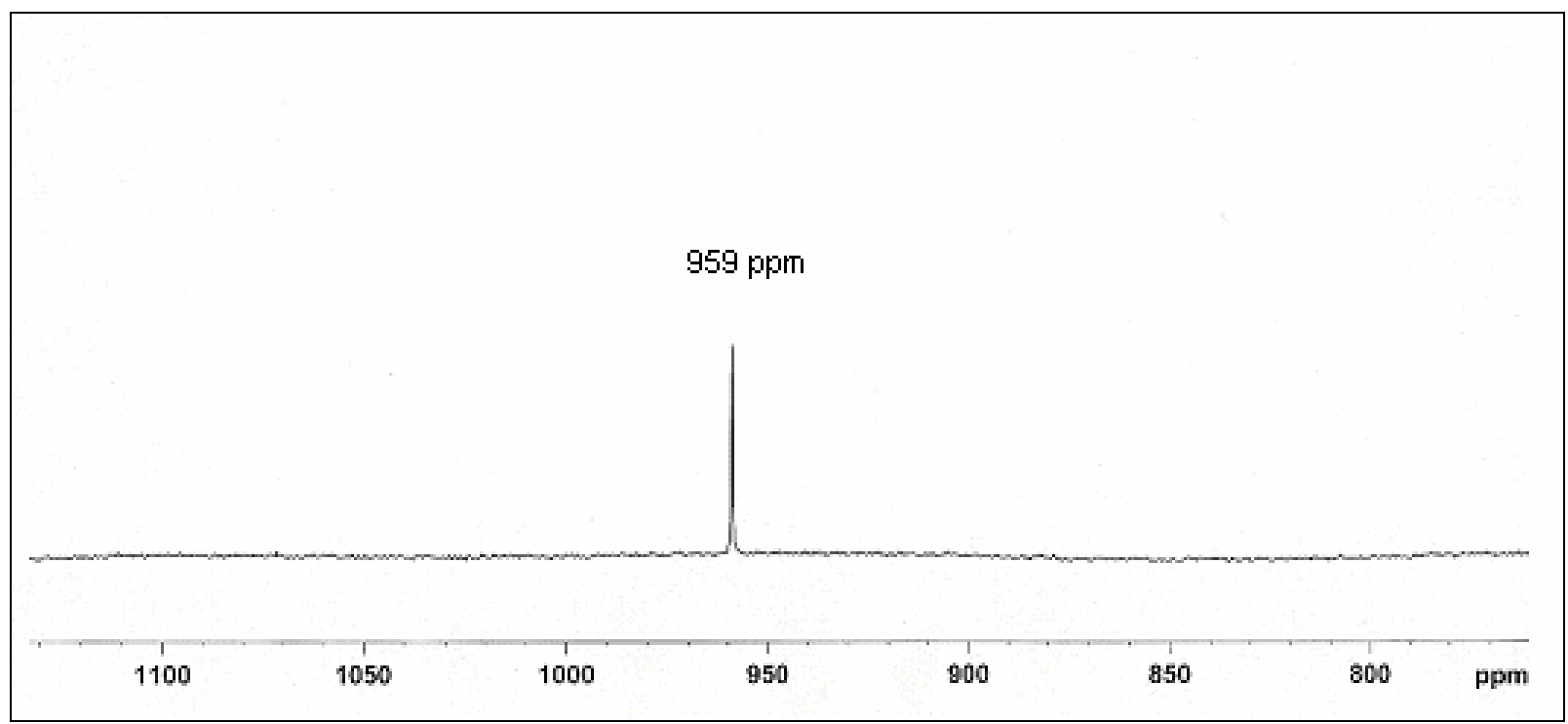


Figure S2. Addition of 1 equiv of $\mathrm{PhSH}$ to ebselen in $\mathrm{CDCl}_{3}$.

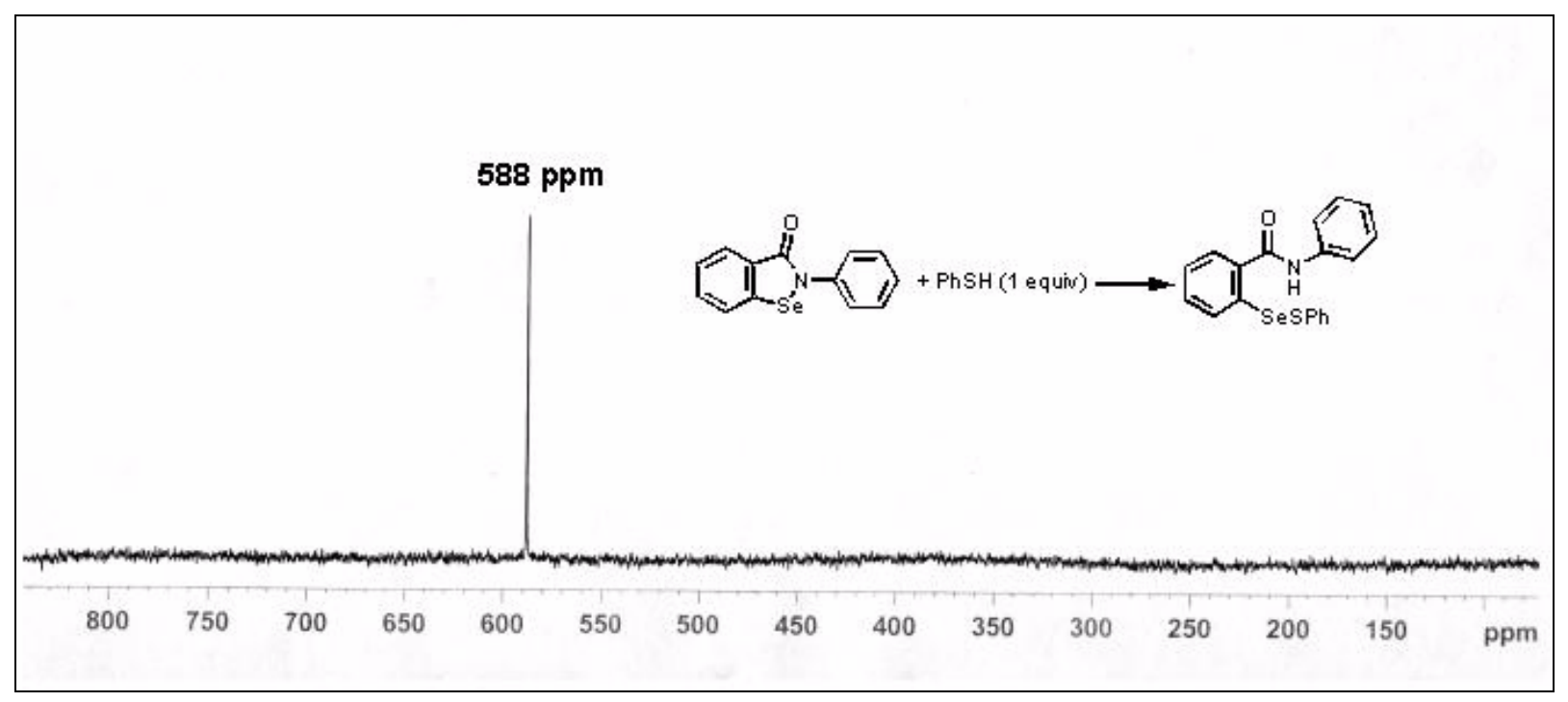

Figure S3. Addition of an excess amount of $\mathrm{PhSH}$ to ebselen in $\mathrm{CDCl}_{3}$.

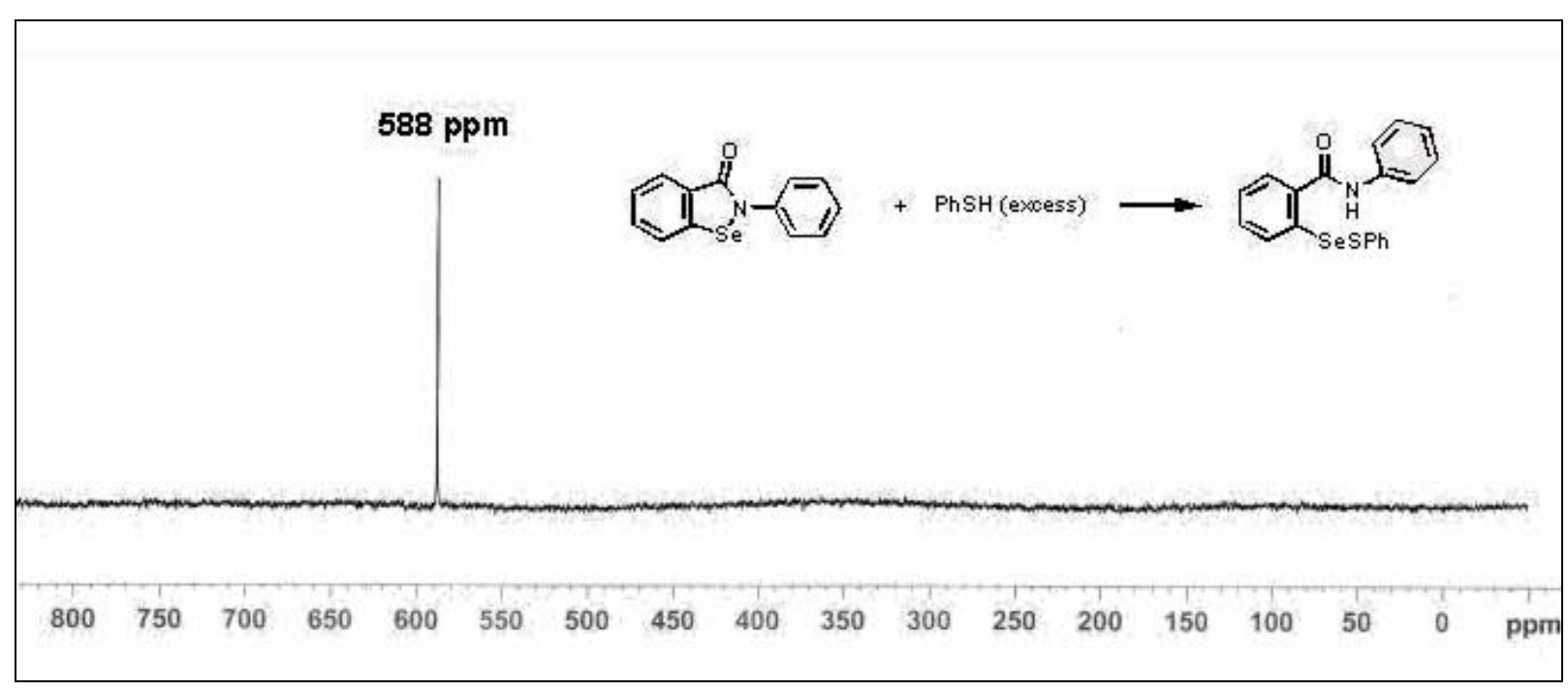


Figure S4. Addition of 1 equiv of $\mathrm{N}$-acetyl-L-Cysteine to ebselen in $\mathrm{CDCl}_{3} / \mathrm{MeOH}$

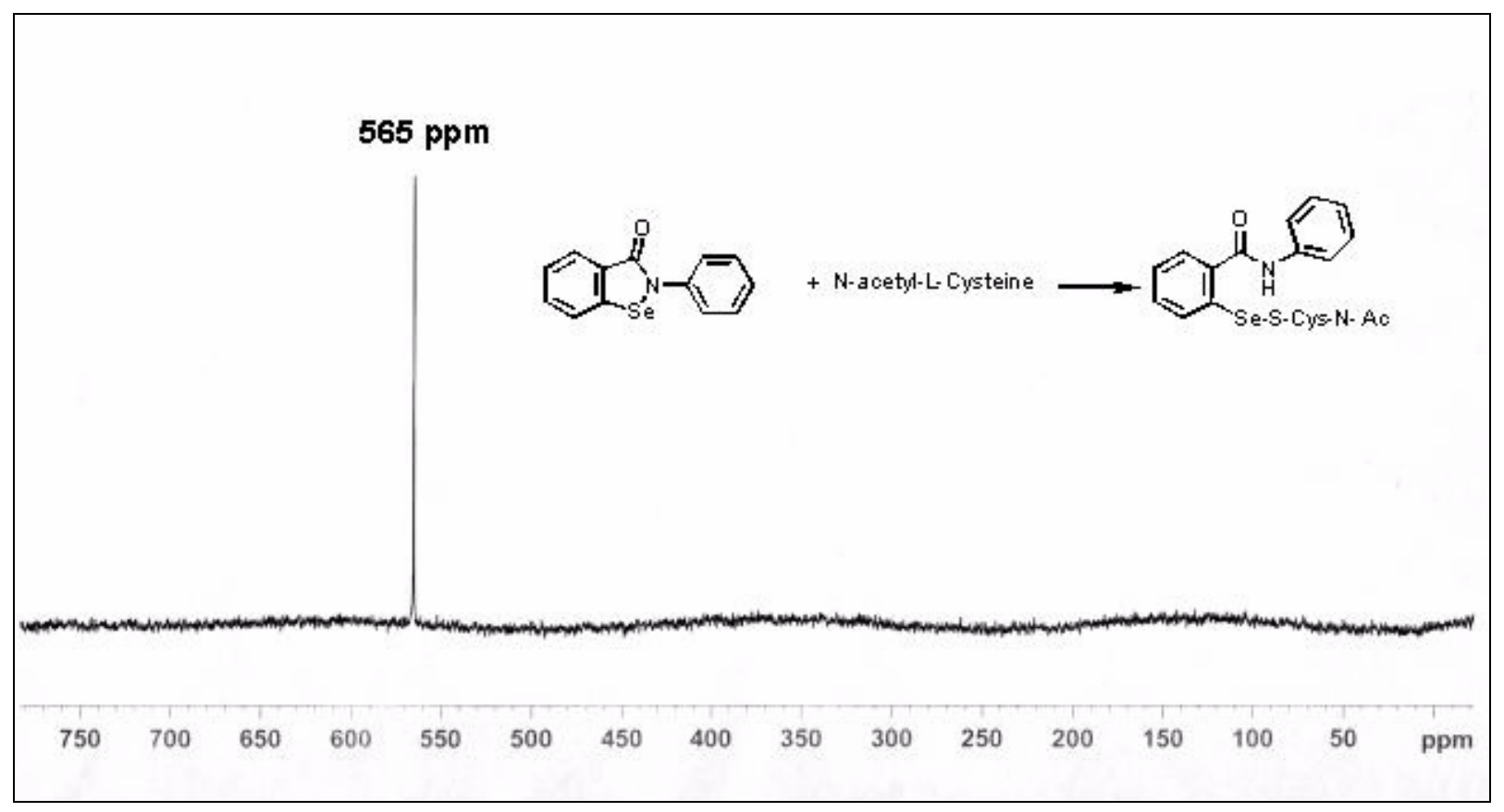

Figure S5. Addition of an excess amount of N-acetyl-L-cysteine to ebselen in $\mathrm{CDCl}_{3} / \mathrm{MeOH}$.

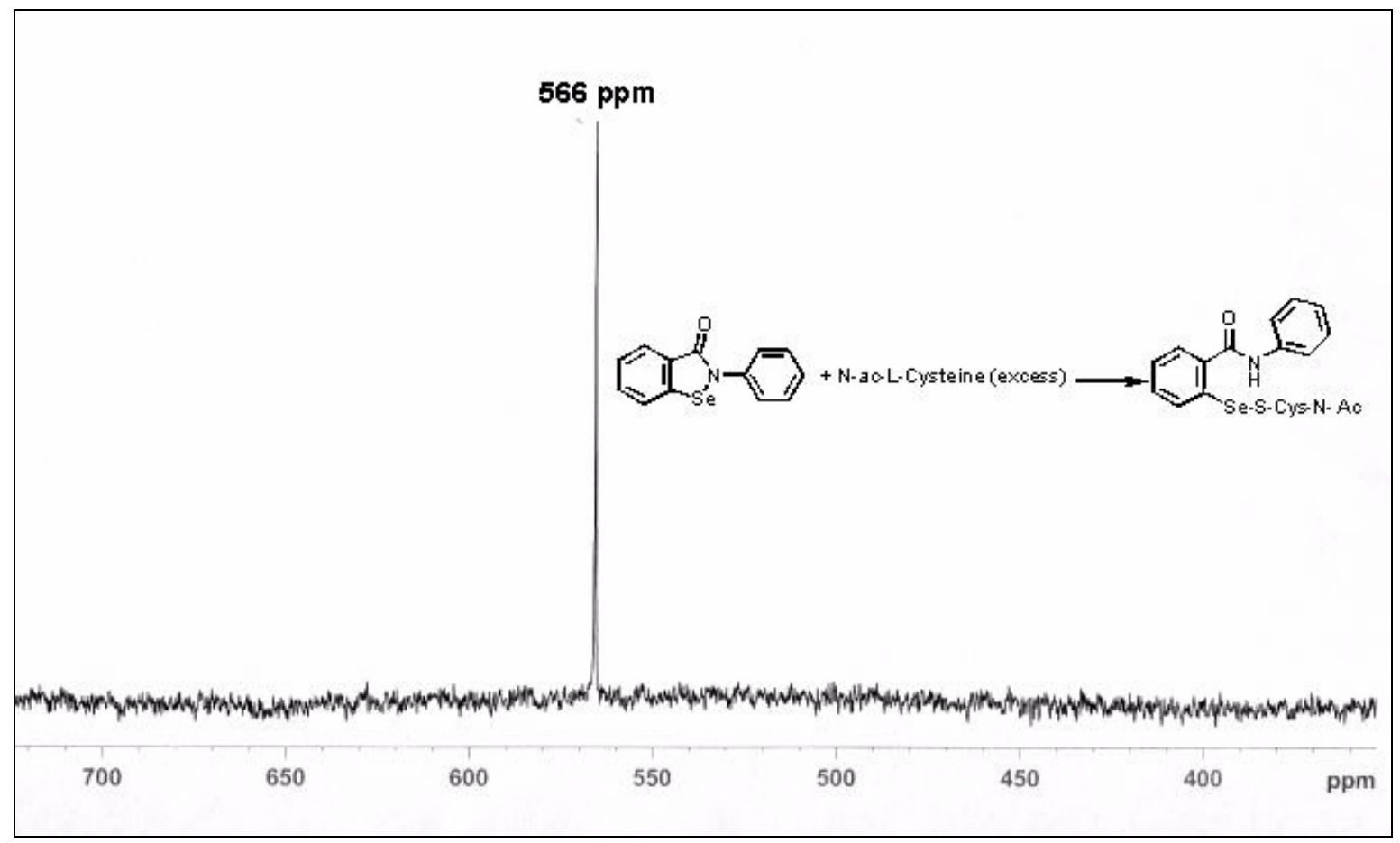


Figure S6. Addition of 1 equiv of N-acetyl-L-cysteine to compound 2 in $\mathrm{CDCl}_{3} / \mathrm{MeOH}$.

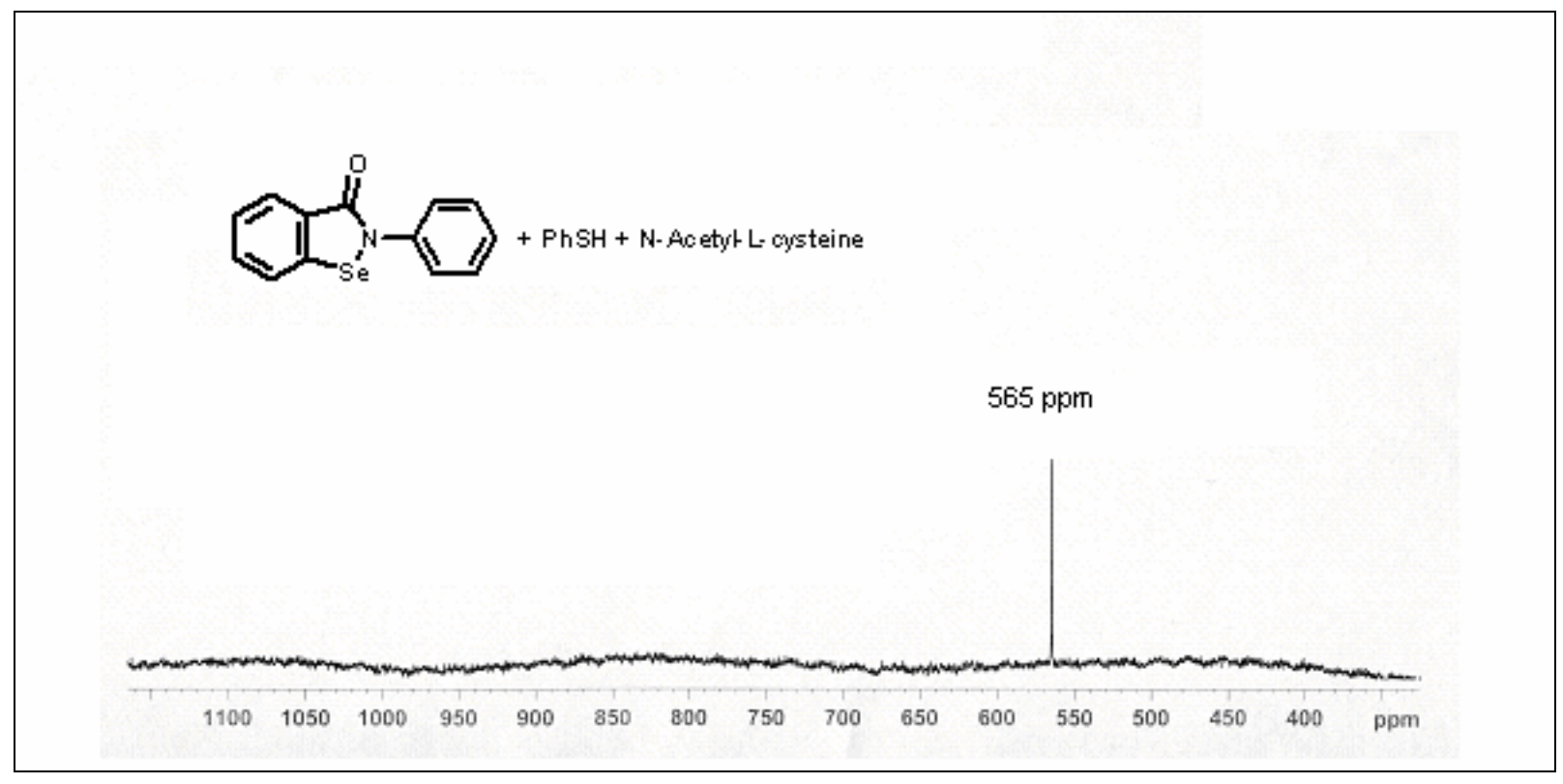

Figure S7. ${ }^{77}$ Se NMR spectrum of pure 9 in $\mathrm{CDCl}_{3}$.

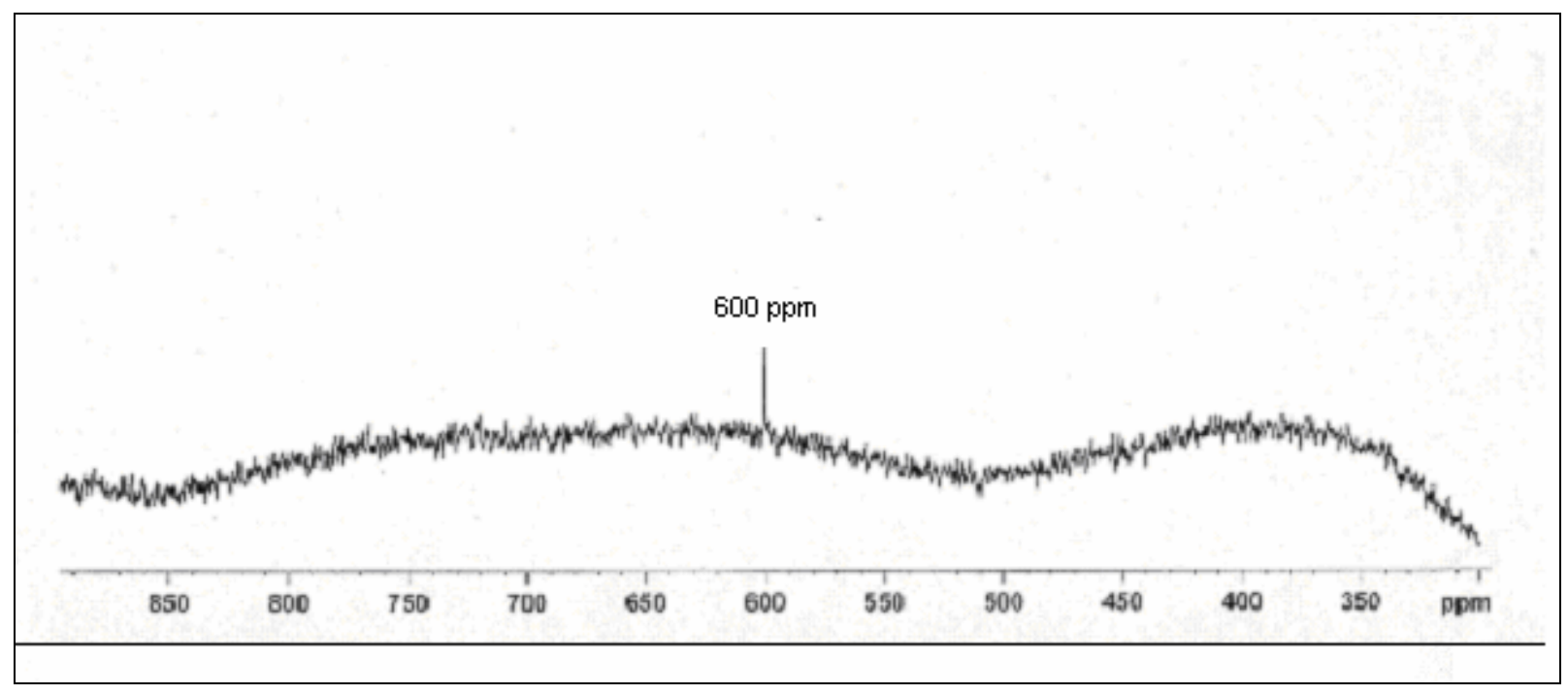


Figure S8. ${ }^{77}$ Se NMR spectrum of pure 19 in $\mathrm{CDCl}_{3}$.

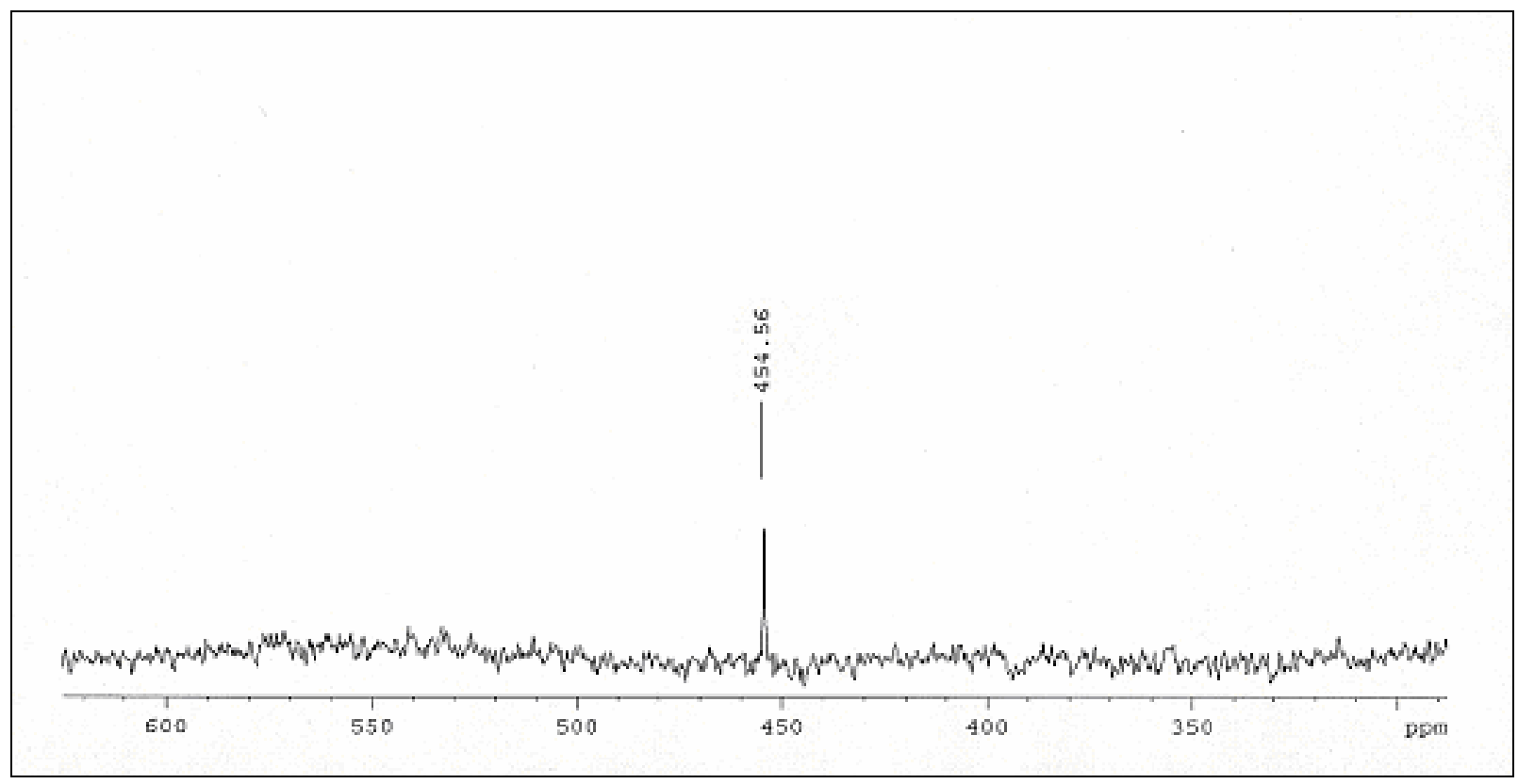

Figure S9. ${ }^{77}$ Se NMR spectrum of pure 24 in $\mathrm{CDCl}_{3}$.

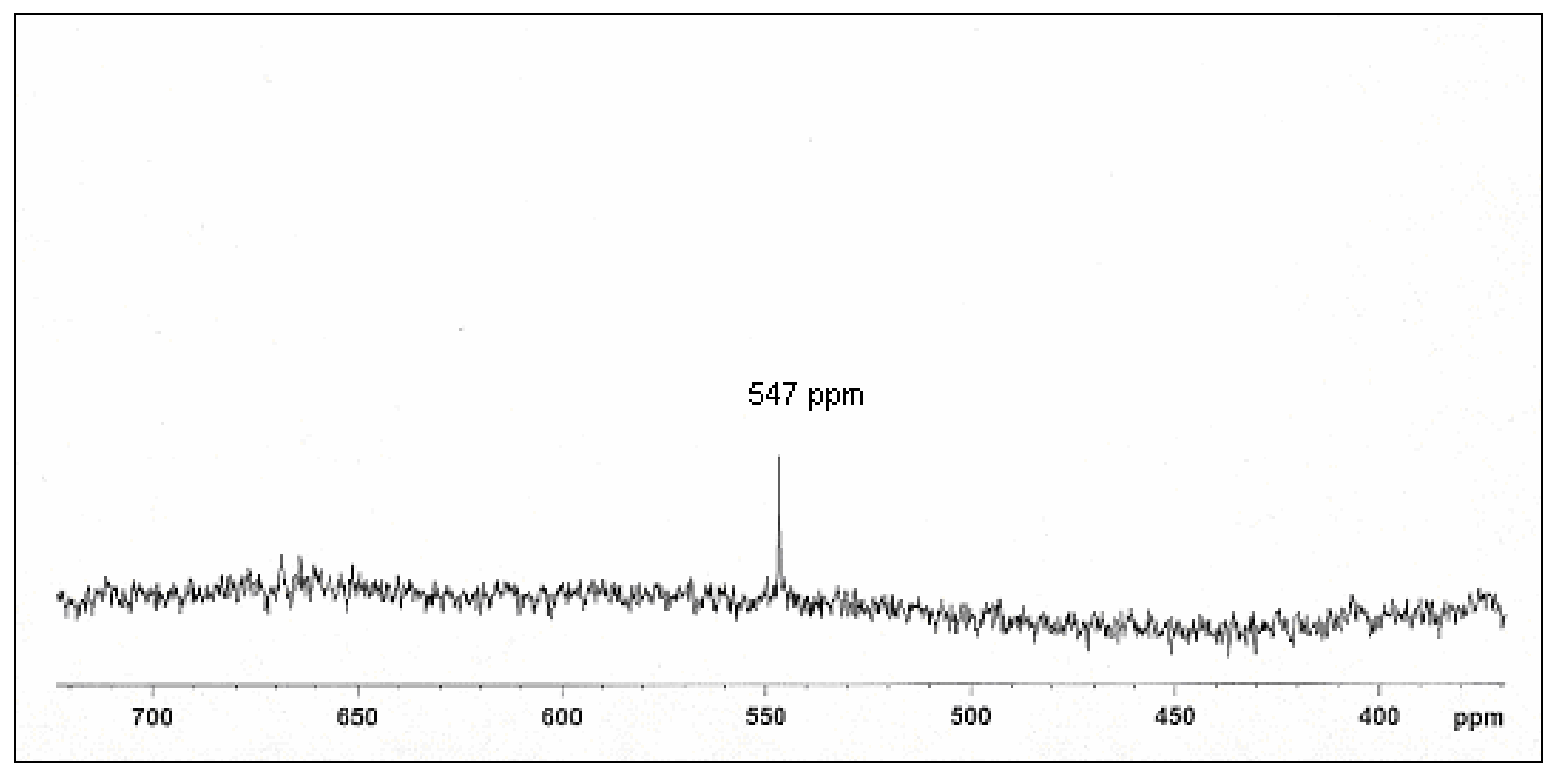


Figure S10. ${ }^{77}$ Se NMR spectrum of 25 in DMSO- $\mathrm{d}_{6}$ produced on addition of excess of thiol 23 to ebselen (1)




Figure S11. ${ }^{77}$ Se NMR spectra and HPLC chromatograms (insert) obtained from the reactions of ebselen with various thiols. (a) reaction of ebselen with an excess of $\mathrm{PhSH}$, (b) reaction of ebselen with an excess of $\mathrm{N}$-acetyl-L-cysteine, (c) reaction of $\mathbf{8}$ with $\mathrm{PhSH}$, (d) reaction of $\mathbf{2}$ with $\mathrm{N}$-acetyl-L-cysteine

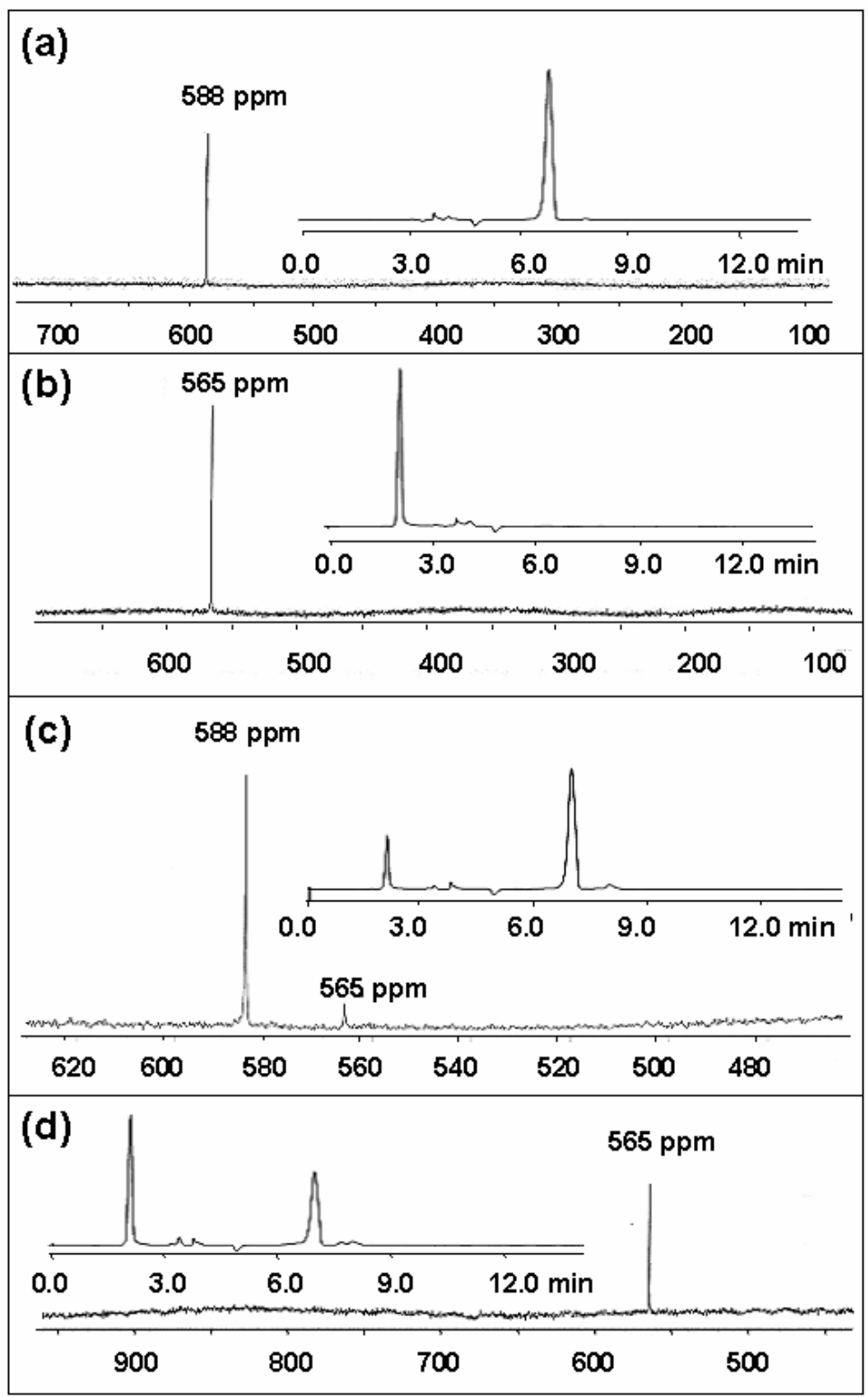


Figure S12. Reaction of ebselen with thiol 23. (a) ${ }^{77}$ Se NMR spectrum of ebselen (b) Addition of one equivalent of 23 to ebselen produced a signal at $547 \mathrm{ppm}$ for selenenyl sulfide 24. (c) The signal at $547 \mathrm{ppm}$ disappeared completely upon addition of an excess of 23 and produced a new signal at 440 ppm for diselenide 25.

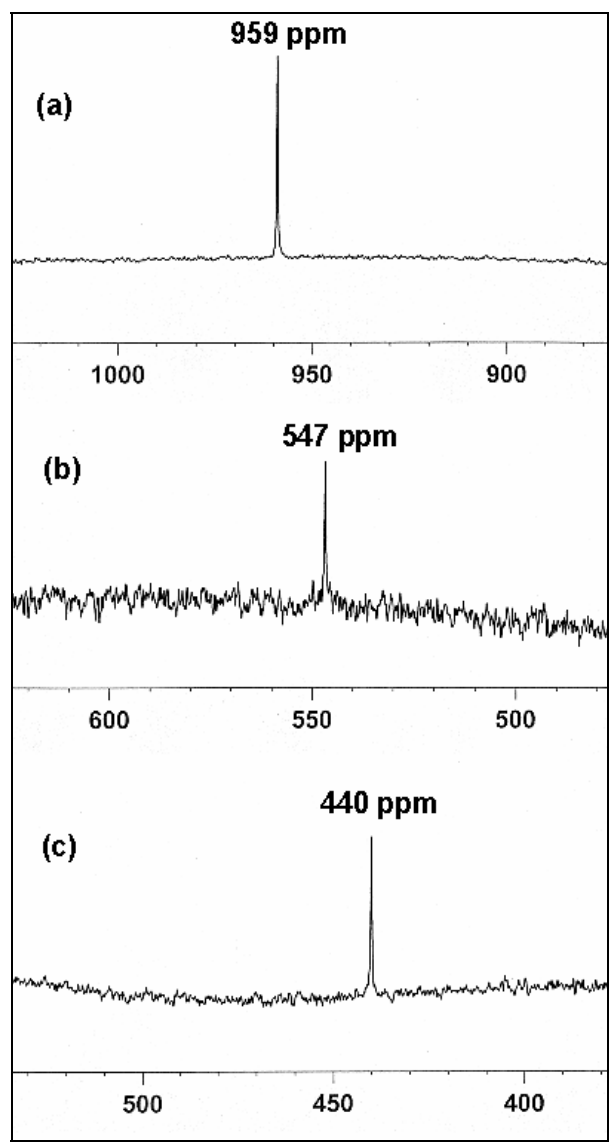


Figure S13. Plot of formation of $\mathrm{PhSSPh}(\mu \mathrm{g})$ with time at various substrate $(\mathrm{PhSH})$ concentrations. The substrate concentration was increased from $3 \mathrm{mM}$ to $27 \mathrm{mM}$. The concentrations of ebselen and $\mathrm{H}_{2} \mathrm{O}_{2}$ were fixed to $0.47 \mathrm{mM}$ and $9.8 \mathrm{mM}$, respectively.
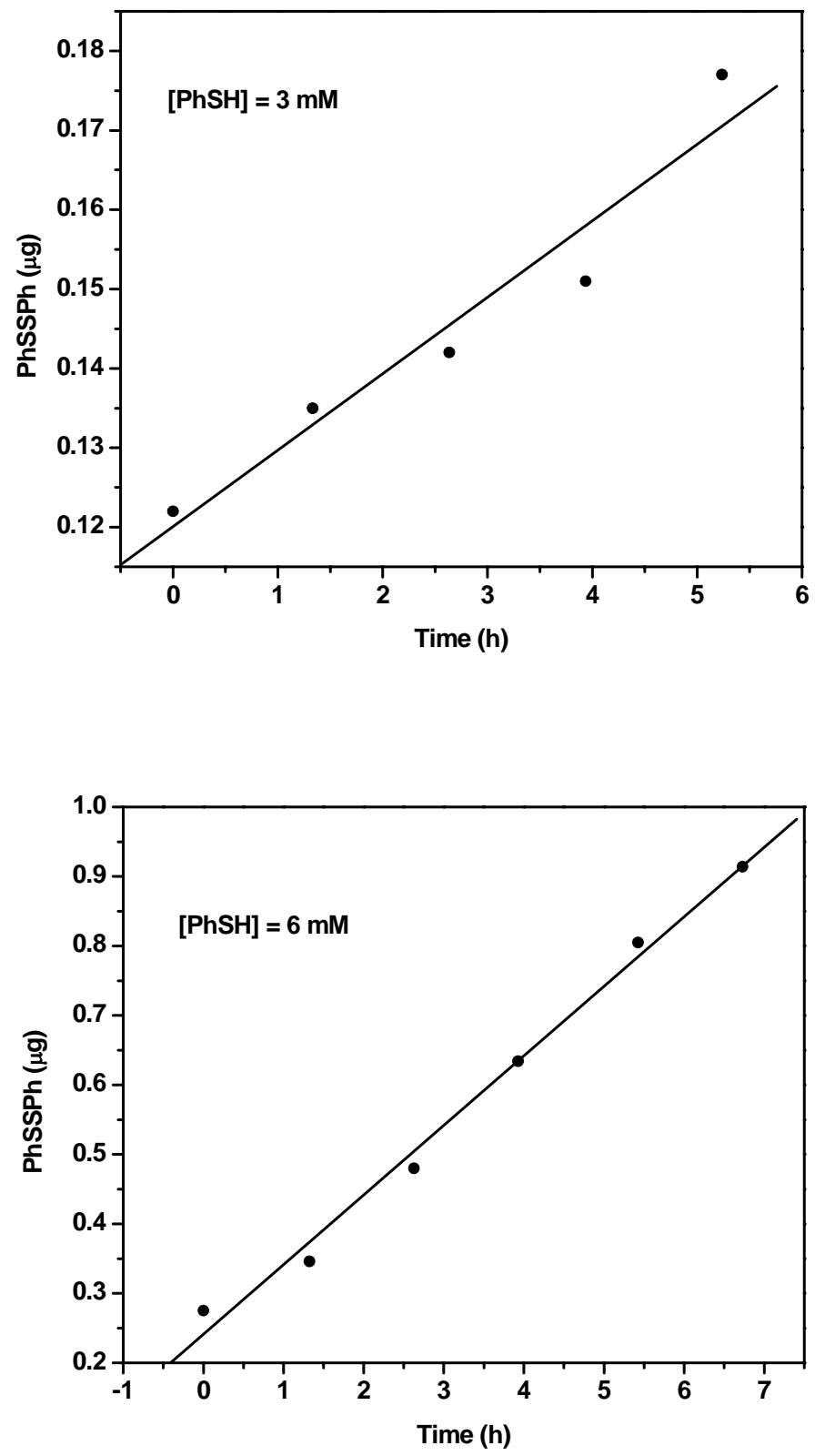

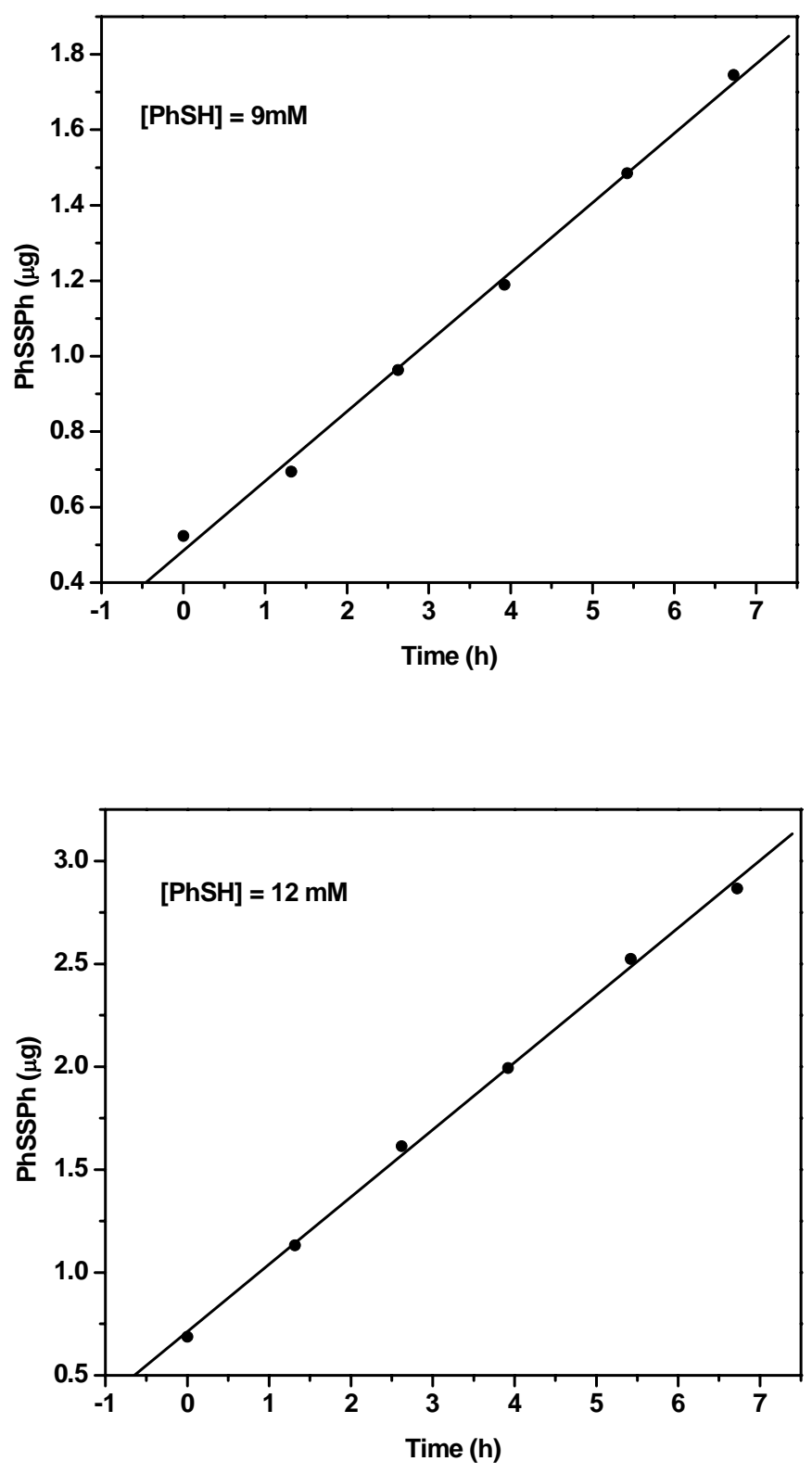

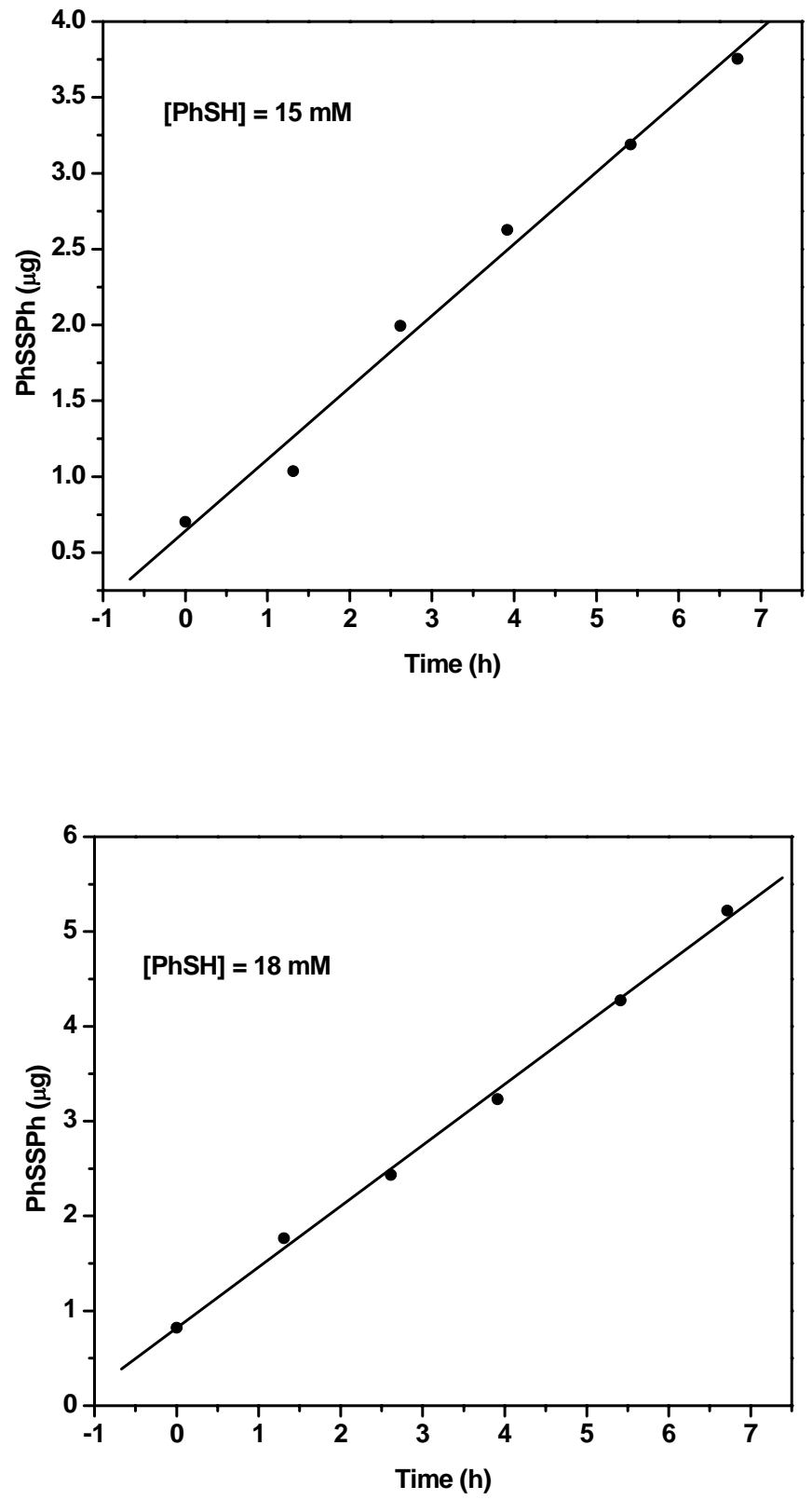

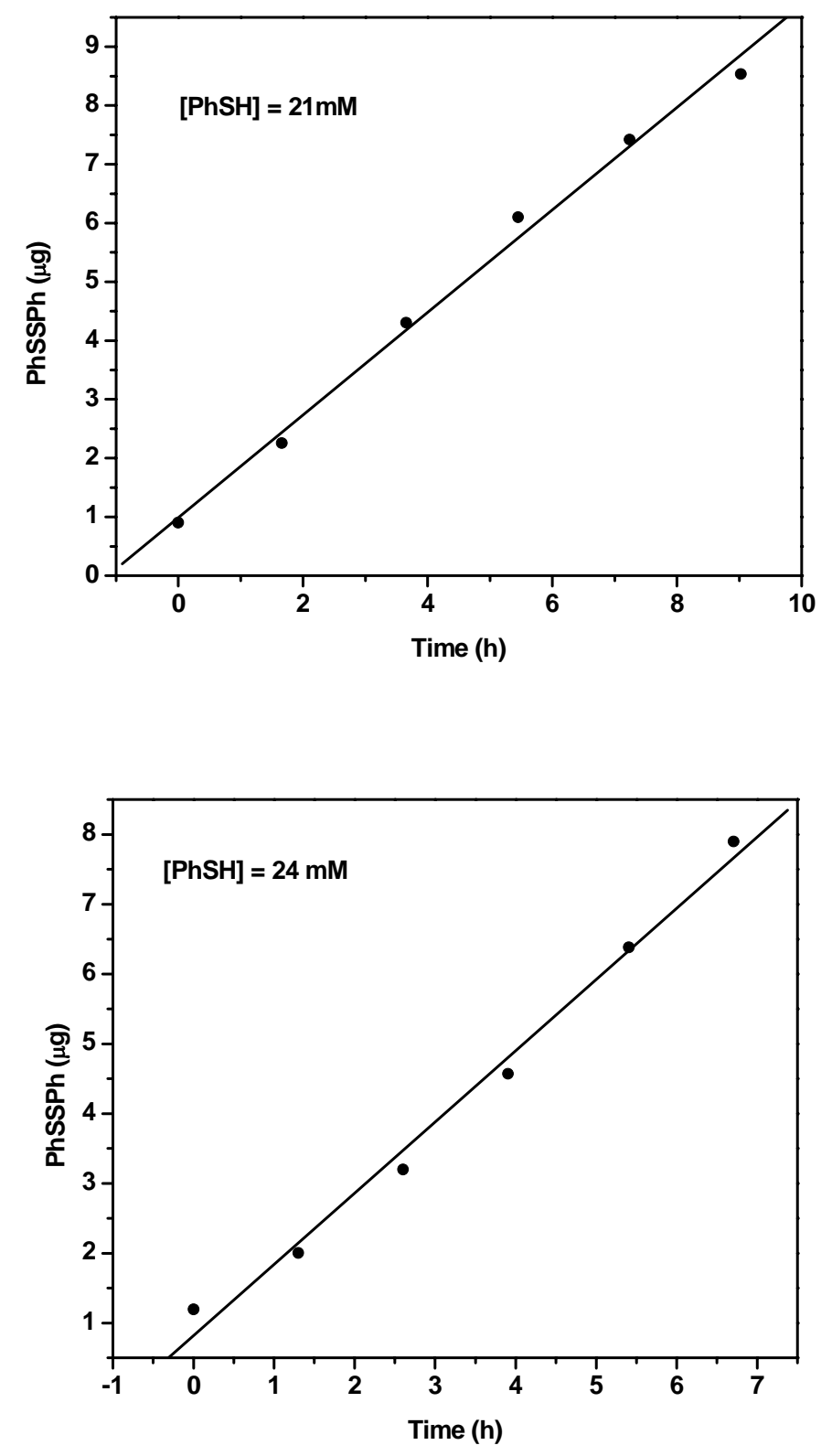


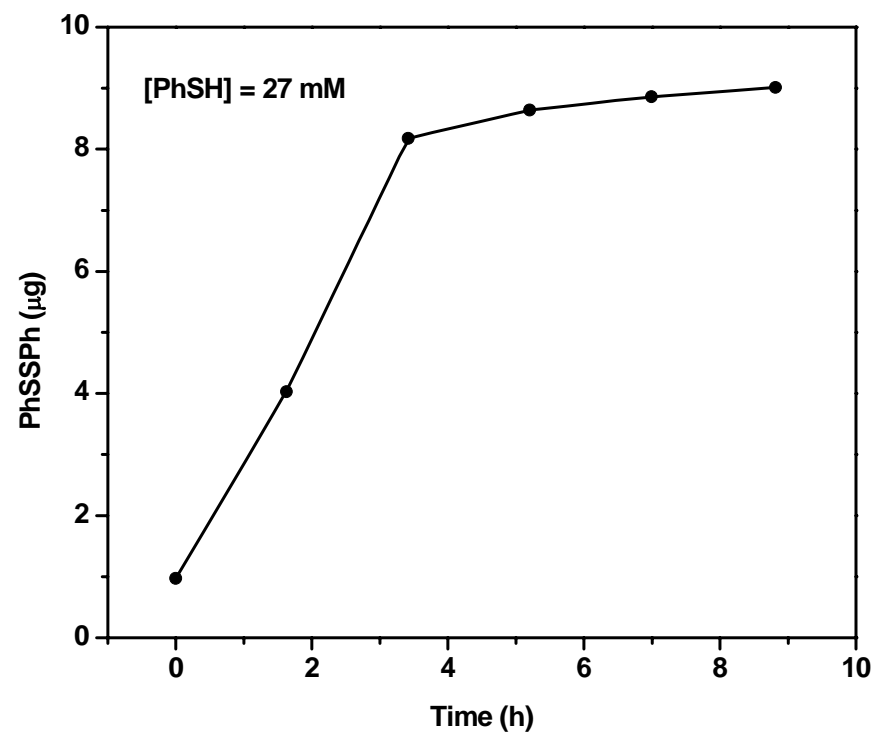

\section{Computational Methods}

All calculations were performed using Gaussian98 suite of quantum chemical S1

programs. Accuracy of the calculated results was confirmed by good agreement between the calculated molecular geometry of $\mathbf{1 3}$ with that determined by X-ray analysis. Table S1 compares the structural parameters of 13. All atomic distances and angles of $\mathbf{1 3}$ in the solid-state are reasonably reproduced by the theoretical calculation at B3LYP/6-31G(d) level within the experimental errors. The fact that the computed structures do not take into account the solid-state packing effects could be taken as the reason for the minor deviations in some of the geometrical parameters compared to the X-ray structure. The ${ }^{77}$ Se NMR chemical shift (547 ppm) calculated at B3LYP/6$311+G(d, p)$ level on B3LYP/6-31G(d) level optimized geometry is found to be very close to the experimental value (575 ppm) for this selenenyl sulfide. 


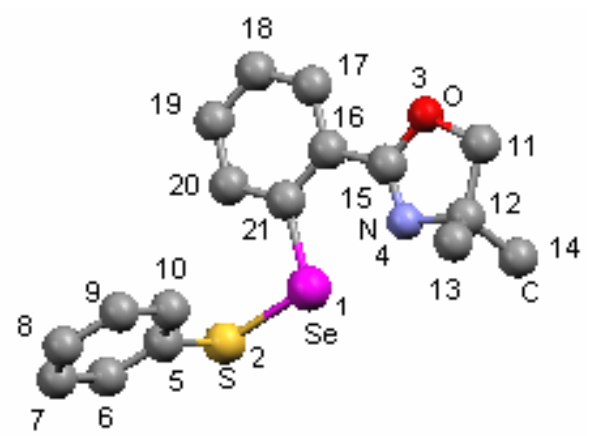

13

Table S1. Structural parameters for [2-(4,4-dimethyl-2-oxazolinylphenyl] selenenyl phenyl sulfide (13) obtained by Quantum Chemical Calculations in comparison to those determined by X-ray Crystallographic Analysis.

\begin{tabular}{|c|c|c|c|}
\hline Atomic distance & B3LYP/6-31G(d)( () & X-ray $(\AA)$ & Difference $(\AA)$ \\
\hline Se-S & 2.250 & 2.218 & 0.032 \\
\hline Se $\cdots \mathrm{N}$ & 2.608 & 2.617 & -0.009 \\
\hline Se-C & 1.949 & 1.943 & 0.006 \\
\hline S-C & 1.805 & 1.786 & 0.019 \\
\hline $\mathrm{C}=\mathrm{N}$ & 1.280 & 1.263 & 0.017 \\
\hline $\mathrm{C}-\mathrm{N}_{12}$ & 1.477 & 1.493 & -0.016 \\
\hline $\mathrm{C}_{15}-\mathrm{O}$ & 1.361 & 1.370 & -0.009 \\
\hline $\mathrm{C}_{11}-\mathrm{O}$ & 1.450 & 1.457 & -0.007 \\
\hline $\mathrm{C}_{15}-\mathrm{C}_{16}$ & 1.457 & 1.462 & -0.005 \\
\hline $\mathrm{C}_{11}-\mathrm{C}_{12}$ & 1.563 & 1.536 & 0.027 \\
\hline $\mathrm{C}_{12}-\mathrm{C}_{14}$ & 1.534 & 1.515 & 0.019 \\
\hline $\mathrm{C}_{12}-\mathrm{C}_{13}$ & 1.536 & 1.519 & 0.017 \\
\hline$C=-C^{a}$ & 1.399 & 1.387 & 0.012 \\
\hline$C=-C^{b}$ & 1.397 & 1.383 & 0.014 \\
\hline Angle & B3LYP/6-31G(d) $\left(^{\circ}\right)$ & X-ray $\left(^{\circ}\right)$ & Difference $\left(^{\circ}\right)$ \\
\hline S-Se $\cdots N$ & 177.5 & 176.6 & 0.9 \\
\hline $\mathrm{C}_{21}$-Se-S & 101.1 & 100.7 & 0.4 \\
\hline
\end{tabular}




\begin{tabular}{|c|c|c|c|}
\hline $\mathrm{C}_{5}-\mathrm{S}-\mathrm{Se}$ & 105.7 & 104.6 & 1.1 \\
\hline $\mathrm{N} \cdots$ Se-C 21 & 76.6 & 76.4 & 0.2 \\
\hline $\mathrm{C}_{16}-\mathrm{C}_{15}-\mathrm{N}$ & 123.6 & 124.3 & -0.7 \\
\hline $\mathrm{C}_{16}-\mathrm{C}_{21}-\mathrm{Se}$ & 119.1 & 119.5 & -0.4 \\
\hline $\mathrm{C}_{20}-\mathrm{C}_{21}-\mathrm{Se}$ & 122.3 & 121.4 & 0.9 \\
\hline $\mathrm{O}-\mathrm{C}_{15}-\mathrm{N}$ & 117.9 & 118.0 & -0.1 \\
\hline $\mathrm{O}-\mathrm{C}_{11}-\mathrm{C}_{12}$ & 105.0 & 104.8 & 0.2 \\
\hline $\mathrm{N}-\mathrm{C}_{12}-\mathrm{C}_{11}$ & 102.4 & 101.8 & 0.6 \\
\hline $\mathrm{O}-\mathrm{C}_{15}-\mathrm{C}_{16}$ & 118.5 & 117.6 & 0.8 \\
\hline $\mathrm{C}_{11}-\mathrm{O}-\mathrm{C}_{15}$ & 105.8 & 104.2 & 1.6 \\
\hline $\mathrm{C}_{12}-\mathrm{N}-\mathrm{C}_{15}$ & 108.4 & 107.7 & 0.7 \\
\hline $\mathrm{S}-\mathrm{C}_{5}-\mathrm{C}_{10}$ & 124.0 & 122.8 & 1.2 \\
\hline $\mathrm{S}-\mathrm{C}_{5}-\mathrm{C}_{6}$ & 116.3 & 117.1 & -0.8 \\
\hline
\end{tabular}

${ }^{\mathrm{a}} \mathrm{Ph}$ ring attached to Se. ${ }^{\mathrm{b}} \mathrm{Ph}$ ring attached to $\mathrm{S}$. 
Table S2. The calculated ${ }^{77}$ Se NMR Chemical Shift and the NBO second-order perturbation energy of 1-7, 13-21, and 26-27.

\begin{tabular}{lll}
\hline Compound & Calc. ${ }^{77}$ Se NMR $(\delta)(\mathrm{ppm})$ & $\mathrm{E}_{\mathrm{Se} / \cdots \mathrm{N} / \mathrm{O}}(\mathrm{kcal} / \mathrm{mol})$ \\
\hline $\mathbf{1}$ & 941 & - \\
$\mathbf{2}$ & 604 & 19.01 \\
$\mathbf{3}$ & 203 & 7.29 \\
$\mathbf{4}$ & 1110 & 28.05 \\
$\mathbf{5}$ & 81 & 5.19 \\
$\mathbf{7}$ & 253 & 6.13 \\
$\mathbf{1 3}$ & 547 & 13.45 \\
$\mathbf{1 4}$ & 197 & 6.35 \\
$\mathbf{1 5}$ & 1021 & 23.01 \\
$\mathbf{1 6}$ & 1052 & 21.64 \\
$\mathbf{1 7}$ & 525 & 15.49 \\
$\mathbf{1 8}$ & 405 & 5.47 \\
$\mathbf{1 9}$ & 405 & 5.46 \\
$\mathbf{2 0}$ & 500,331 & 12.61 \\
$\mathbf{2 1}$ & 460 & 4.88 \\
$\mathbf{2 6}$ & 632 & 14.35 \\
$\mathbf{2 7}$ & 585 & $11.43,4.78$ \\
\hline
\end{tabular}


Figure S14. Optimized structures for the model selenium compounds in gas phase obtained by quantum chemical calculation at the B3LYP/6-31G(d) level. Bond lengths are given in angstroms.

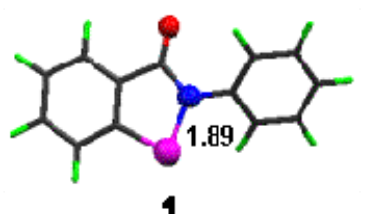

1
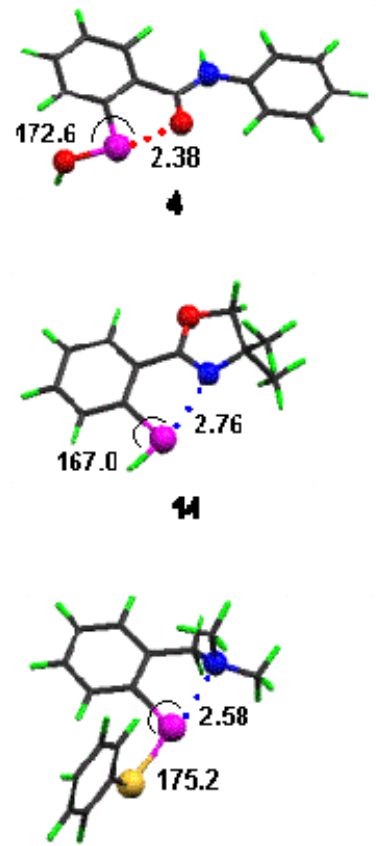

17

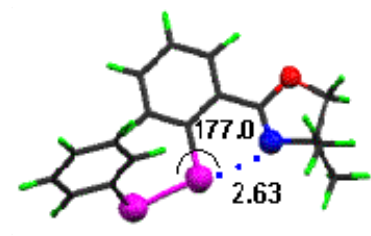

20
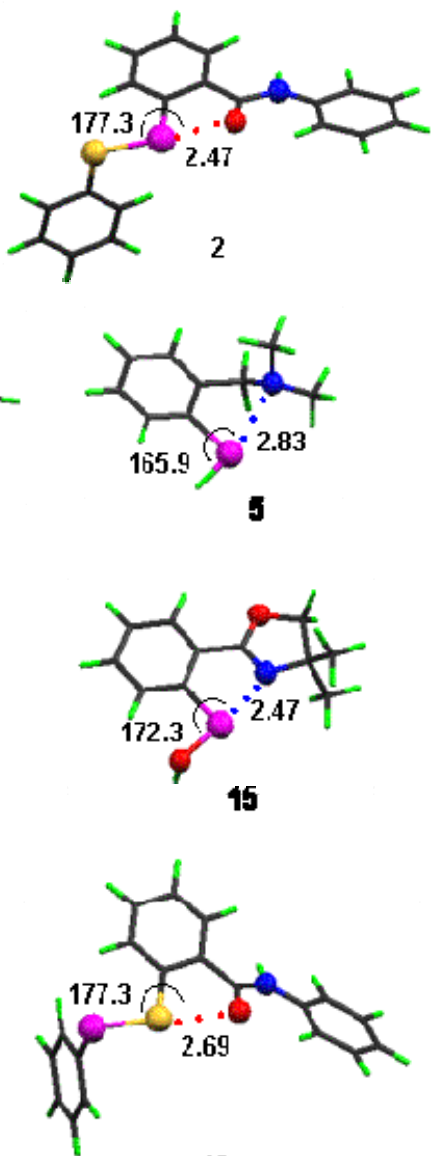

19

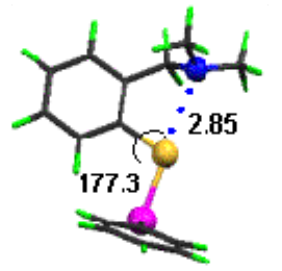

21

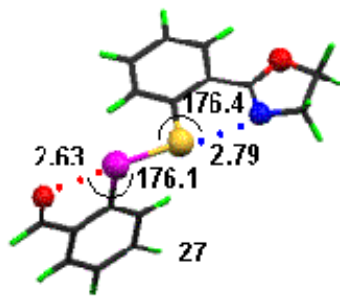

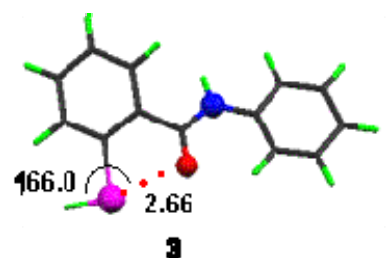
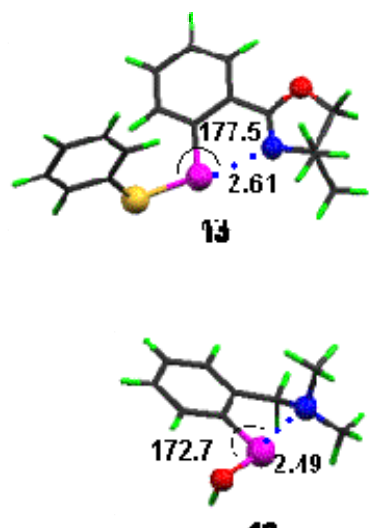

18
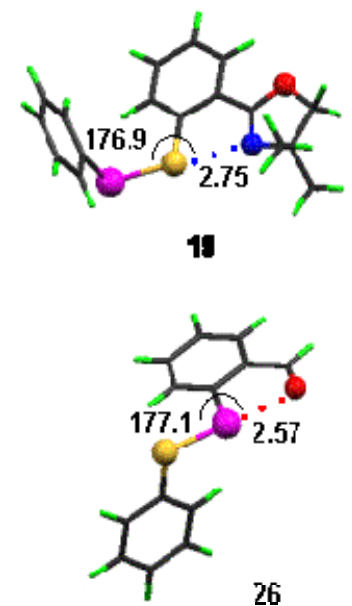
Cartesian Coordinates. The Cartesian coordinates of the optimized compounds are listed in the following pages.

\begin{tabular}{|c|c|c|c|c|c|c|c|}
\hline \multicolumn{4}{|c|}{ Compound $\mathbf{1}$} & \multicolumn{4}{|c|}{ Compound 2} \\
\hline C & -4.226245 & 0.886215 & 0.163892 & & & & \\
\hline C & -4.283444 & -0.382762 & 0.760146 & $\mathrm{~S}$ & -2.228015 & -2.573758 & 0.687767 \\
\hline C & -3.128258 & -1.137524 & 0.958308 & $\mathrm{Se}$ & -0.775239 & -0.898887 & 1.066707 \\
\hline C & -1.908803 & -0.597608 & 0.549859 & $\mathrm{O}$ & 0.790291 & 0.986134 & 1.380631 \\
\hline C & -1.839067 & 0.664946 & -0.044086 & $\mathrm{~N}$ & 1.402775 & 2.837980 & 0.182795 \\
\hline C & -3.003661 & 1.412459 & -0.239516 & $\mathrm{C}$ & -1.366860 & 0.411223 & -0.242055 \\
\hline Se & -0.196577 & -1.385710 & 0.694615 & C & -0.639152 & 1.615988 & -0.382949 \\
\hline $\mathrm{N}$ & 0.468737 & 0.176027 & -0.142890 & $\mathrm{C}$ & -1.092230 & 2.587389 & -1.293018 \\
\hline C & -0.495741 & 1.140343 & -0.440644 & $\mathrm{H}$ & -0.583466 & 3.544372 & -1.377407 \\
\hline $\mathrm{O}$ & -0.250547 & 2.220766 & -0.963773 & $\mathrm{C}$ & -2.218183 & 2.372876 & -2.076103 \\
\hline C & 1.860105 & 0.285136 & -0.401838 & $\mathrm{H}$ & -2.554431 & 3.137785 & -2.769425 \\
\hline C & 2.595666 & -0.866448 & -0.717333 & $\mathrm{C}$ & -2.912879 & 1.167099 & -1.952301 \\
\hline C & 3.967772 & -0.782591 & -0.945750 & $\mathrm{H}$ & -3.794626 & 0.980510 & -2.559316 \\
\hline C & 4.617473 & 0.450450 & -0.885092 & C & -2.493559 & 0.201454 & -1.042859 \\
\hline C & 3.880254 & 1.597972 & -0.587088 & $\mathrm{H}$ & -3.051558 & -0.723158 & 0.944648 \\
\hline C & 2.510665 & 1.527111 & -0.339304 & C & 0.569925 & 1.790802 & 0.464241 \\
\hline $\mathrm{H}$ & -5.139214 & 1.455953 & 0.018591 & $\mathrm{C}$ & 2.593697 & 3.235626 & 0.832959 \\
\hline $\mathrm{H}$ & -5.241609 & -0.788349 & 1.073406 & C & 3.097239 & 2.614376 & 1.985589 \\
\hline $\mathrm{H}$ & -3.185444 & -2.119097 & 1.419807 & $\mathrm{H}$ & 2.571394 & 1.774705 & 2.416225 \\
\hline $\mathrm{H}$ & -2.921413 & 2.390164 & -0.704876 & $\mathrm{C}$ & 4.278203 & 3.091563 & 2.555064 \\
\hline $\mathrm{H}$ & 2.088101 & -1.824204 & -0.796466 & $\mathrm{H}$ & 4.661460 & 2.604106 & 3.447467 \\
\hline $\mathrm{H}$ & 4.524444 & -1.684706 & -1.184265 & $\mathrm{C}$ & 4.966621 & 4.171389 & 2.001686 \\
\hline $\mathrm{H}$ & 5.685940 & 0.516992 & -1.069240 & $\mathrm{H}$ & 5.885122 & 4.530156 & 2.456860 \\
\hline $\mathrm{H}$ & 4.374510 & 2.564514 & -0.538163 & $\mathrm{C}$ & 4.460813 & 4.785704 & 0.854576 \\
\hline \multirow[t]{15}{*}{$\mathrm{H}$} & 1.941320 & 2.418349 & -0.114600 & $\mathrm{H}$ & 4.981097 & 5.628179 & 0.407292 \\
\hline & & & & $\mathrm{C}$ & 3.283780 & 4.323223 & 0.274161 \\
\hline & & & & $\mathrm{H}$ & 2.894163 & 4.807878 & -0.619526 \\
\hline & & & & C & -1.513625 & -3.541727 & -0.658433 \\
\hline & & & & C & -0.331444 & -3.201277 & -1.321560 \\
\hline & & & & $\mathrm{H}$ & 0.213065 & -2.312791 & -1.018139 \\
\hline & & & & C & 0.143735 & -4.012381 & -2.353157 \\
\hline & & & & $\mathrm{H}$ & 1.065319 & -3.739044 & -2.860977 \\
\hline & & & & $\mathrm{C}$ & -0.550433 & -5.161929 & -2.732012 \\
\hline & & & & $\mathrm{H}$ & -0.175976 & -5.789163 & -3.536192 \\
\hline & & & & $\mathrm{C}$ & -1.730943 & -5.499801 & -2.066573 \\
\hline & & & & $\mathrm{H}$ & -2.281120 & -6.393616 & -2.349478 \\
\hline & & & & C & -2.214206 & -4.696358 & -1.034563 \\
\hline & & & & $\mathrm{H}$ & -3.133150 & -4.965643 & -0.519960 \\
\hline & & & & $\mathrm{H}$ & 1.184347 & 3.371634 & -0.645860 \\
\hline
\end{tabular}




\begin{tabular}{|c|c|c|c|c|c|c|c|}
\hline \multicolumn{4}{|c|}{ Compound 3} & \multicolumn{4}{|c|}{ Compound 4} \\
\hline Se & -0.989706 & -2.570054 & 1.060442 & Se & -1.674671 & -0.964510 & 1.712724 \\
\hline $\mathrm{O}$ & 0.861827 & -0.773550 & 0.432454 & $\mathrm{O}$ & 0.447581 & -0.555574 & 0.728063 \\
\hline $\mathrm{N}$ & 0.612144 & 1.201301 & -0.705370 & $\mathrm{~N}$ & 1.347208 & 0.544232 & -1.068206 \\
\hline $\mathrm{C}$ & -1.913159 & -0.893324 & 0.995790 & $\mathrm{C}$ & -2.088682 & 0.188824 & 0.244005 \\
\hline $\mathrm{C}$ & -1.261705 & 0.278359 & 0.549900 & $\mathrm{C}$ & -1.056954 & 0.646052 & -0.605339 \\
\hline $\mathrm{C}$ & -1.955066 & 1.501296 & 0.578302 & $\mathrm{C}$ & -1.374370 & 1.500399 & -1.676608 \\
\hline $\mathrm{H}$ & -1.441264 & 2.420438 & 0.309427 & $\mathrm{H}$ & -0.599562 & 1.874287 & -2.342156 \\
\hline $\mathrm{C}$ & -3.277966 & 1.577801 & 0.994585 & $\mathrm{C}$ & -2.683365 & 1.896781 & -1.909058 \\
\hline $\mathrm{H}$ & -3.790373 & 2.534801 & 1.015670 & $\mathrm{H}$ & -2.913402 & 2.557685 & -2.739184 \\
\hline $\mathrm{C}$ & -3.930540 & 0.409984 & 1.397036 & $\mathrm{C}$ & -3.699532 & 1.441487 & -1.058837 \\
\hline $\mathrm{H}$ & -4.966284 & 0.445776 & 1.723775 & $\mathrm{H}$ & -4.727070 & 1.752749 & -1.228161 \\
\hline $\mathrm{C}$ & -3.252851 & -0.804052 & 1.403902 & $\mathrm{C}$ & -3.407323 & 0.599110 & 0.006322 \\
\hline $\mathrm{H}$ & -3.768716 & -1.697701 & 1.741051 & $\mathrm{H}$ & -4.186128 & 0.256436 & 0.678020 \\
\hline $\mathrm{C}$ & 0.160576 & 0.183747 & 0.100379 & $\mathrm{C}$ & 0.302345 & 0.172456 & -0.271903 \\
\hline $\mathrm{C}$ & 1.900272 & 1.401354 & -1.248931 & $\mathrm{C}$ & 2.720829 & 0.230475 & -0.956454 \\
\hline $\mathrm{C}$ & 3.003947 & 0.586251 & -0.954227 & $\mathrm{C}$ & 3.259707 & -0.557333 & 0.071837 \\
\hline $\mathrm{H}$ & 2.882810 & -0.258050 & -0.291169 & $\mathrm{H}$ & 2.609976 & -0.961617 & 0.834238 \\
\hline $\mathrm{C}$ & 4.240008 & 0.877021 & -1.532178 & $\mathrm{C}$ & 4.632397 & -0.806011 & 0.091087 \\
\hline $\mathrm{H}$ & 5.088459 & 0.239409 & -1.298274 & $\mathrm{H}$ & 5.040859 & -1.417055 & 0.891513 \\
\hline $\mathrm{C}$ & 4.400767 & 1.959912 & -2.397039 & $\mathrm{C}$ & 5.477982 & -0.288000 & -0.890155 \\
\hline $\mathrm{H}$ & 5.369539 & 2.172334 & -2.839804 & $\mathrm{H}$ & 6.544501 & -0.491013 & -0.861077 \\
\hline $\mathrm{C}$ & 3.299472 & 2.767363 & -2.687452 & $\mathrm{C}$ & 4.936249 & 0.495351 & -1.910978 \\
\hline $\mathrm{H}$ & 3.402086 & 3.615928 & -3.358429 & $\mathrm{H}$ & 5.576772 & 0.908922 & -2.685086 \\
\hline $\mathrm{C}$ & 2.059635 & 2.492508 & -2.118566 & $\mathrm{C}$ & 3.569450 & 0.753462 & -1.945336 \\
\hline $\mathrm{H}$ & 1.206063 & 3.127992 & -2.348433 & $\mathrm{H}$ & 3.154651 & 1.365439 & -2.744447 \\
\hline $\mathrm{H}$ & -2.264899 & -3.320366 & 1.314088 & $\mathrm{O}$ & -3.433499 & -1.152363 & 2.309259 \\
\hline $\mathrm{H}$ & -0.087382 & 1.851244 & -1.033991 & $\mathrm{H}$ & 1.116801 & 1.122757 & -1.862975 \\
\hline & & & & $\mathrm{H}$ & -3.754105 & -1.959681 & 1.874076 \\
\hline
\end{tabular}




\begin{tabular}{|c|c|c|c|c|c|c|c|}
\hline \multicolumn{4}{|c|}{ Compound 5} & \multicolumn{4}{|c|}{ Compound 7} \\
\hline $\mathrm{C}$ & -0.935800 & -0.229825 & -0.240918 & Se & -2.068565 & -1.438135 & 2.042571 \\
\hline $\mathrm{C}$ & -2.274370 & -0.075657 & -0.616627 & $\mathrm{O}$ & 0.144167 & 0.092965 & 1.614302 \\
\hline $\mathrm{C}$ & -2.607104 & 0.315677 & -1.913344 & $\mathrm{~N}$ & 1.064067 & 0.454925 & -0.457313 \\
\hline $\mathrm{C}$ & -1.601838 & 0.578710 & -2.843827 & $\mathrm{C}$ & -2.385825 & -0.517079 & 0.389414 \\
\hline $\mathrm{C}$ & -0.266942 & 0.440749 & -2.464182 & $\mathrm{C}$ & -1.345113 & 0.212922 & -0.235417 \\
\hline $\mathrm{C}$ & 0.083905 & 0.029389 & -1.174620 & $\mathrm{C}$ & -1.611093 & 0.919803 & -1.419124 \\
\hline $\mathrm{C}$ & 1.534146 & -0.167202 & -0.790121 & $\mathrm{H}$ & -0.832517 & 1.536072 & -1.861221 \\
\hline $\mathrm{N}$ & 1.845684 & 0.488084 & 0.479008 & $\mathrm{C}$ & -2.866448 & 0.893080 & -2.016786 \\
\hline $\mathrm{C}$ & 3.065357 & -0.011615 & 1.094950 & $\mathrm{H}$ & -3.052013 & 1.455089 & -2.927120 \\
\hline $\mathrm{C}$ & 1.814788 & 1.942282 & 0.400971 & $\mathrm{C}$ & -3.881516 & 0.146402 & -1.418516 \\
\hline Se & -0.454303 & -0.766301 & 1.546203 & $\mathrm{H}$ & -4.871451 & 0.110855 & -1.865775 \\
\hline $\mathrm{H}$ & -1.762778 & -1.475977 & 1.756229 & $\mathrm{C}$ & -3.643635 & -0.540964 & -0.230900 \\
\hline $\mathrm{H}$ & -3.062093 & -0.261664 & 0.107596 & $\mathrm{H}$ & -4.459458 & -1.094145 & 0.218424 \\
\hline $\mathrm{H}$ & -3.652943 & 0.424605 & -2.188356 & $\mathrm{C}$ & 0.007718 & 0.252507 & 0.402150 \\
\hline $\mathrm{H}$ & -1.852935 & 0.894865 & -3.852392 & $\mathrm{C}$ & 2.439142 & 0.578160 & -0.163124 \\
\hline $\mathrm{H}$ & 0.524376 & 0.648246 & -3.181375 & $\mathrm{C}$ & 2.954222 & 0.608295 & 1.142145 \\
\hline $\mathrm{H}$ & 2.194000 & 0.168304 & -1.612548 & $\mathrm{H}$ & 2.282252 & 0.514772 & 1.982977 \\
\hline $\mathrm{H}$ & 1.716385 & -1.240995 & -0.645408 & $\mathrm{C}$ & 4.329439 & 0.749413 & 1.329717 \\
\hline $\mathrm{H}$ & 3.973942 & 0.207575 & 0.502834 & $\mathrm{H}$ & 4.718415 & 0.771069 & 2.344340 \\
\hline $\mathrm{H}$ & 2.991035 & -1.095786 & 1.228620 & $\mathrm{C}$ & 5.202776 & 0.859567 & 0.247205 \\
\hline $\mathrm{H}$ & 3.186548 & 0.445779 & 2.082563 & $\mathrm{H}$ & 6.271228 & 0.967717 & 0.409913 \\
\hline $\mathrm{H}$ & 1.916481 & 2.361986 & 1.407040 & $\mathrm{C}$ & 4.685664 & 0.827335 & -1.049254 \\
\hline $\mathrm{H}$ & 2.624647 & 2.355140 & -0.230574 & $\mathrm{H}$ & 5.347724 & 0.910726 & -1.906945 \\
\hline $\mathrm{H}$ & 0.856995 & 2.270532 & -0.011890 & $\mathrm{C}$ & 3.316294 & 0.688733 & -1.254424 \\
\hline & & & & $\mathrm{H}$ & 2.920480 & 0.666198 & -2.268342 \\
\hline & & & & $\mathrm{H}$ & 0.844091 & 0.422421 & -1.442683 \\
\hline & & & & $\mathrm{C}$ & -3.670926 & -2.591537 & 2.072681 \\
\hline & & & & $\mathrm{H}$ & -3.747776 & -3.178038 & 1.155386 \\
\hline & & & & $\mathrm{H}$ & -3.510049 & -3.264173 & 2.918792 \\
\hline & & & & $\mathrm{H}$ & -4.581295 & -2.014957 & 2.246926 \\
\hline
\end{tabular}




\begin{tabular}{|lrrr|}
\hline \multicolumn{4}{|c|}{ Compound $\mathbf{1 3}$} \\
Se & 0.143165 & -1.013189 & 0.396159 \\
S & -1.811056 & -2.114354 & 0.223279 \\
$\mathrm{O}$ & 3.419622 & 1.720152 & -0.930498 \\
$\mathrm{~N}$ & 2.411062 & 0.271242 & 0.484125 \\
$\mathrm{C}$ & -3.075812 & -0.953917 & 0.782332 \\
$\mathrm{C}$ & -4.388570 & -1.443571 & 0.835307 \\
$\mathrm{H}$ & -4.596020 & -2.472032 & 0.550637 \\
$\mathrm{C}$ & -5.426659 & -0.612184 & 1.253808 \\
$\mathrm{H}$ & -6.440607 & -1.002216 & 1.291920 \\
$\mathrm{C}$ & -5.168861 & 0.710272 & 1.621479 \\
$\mathrm{H}$ & -5.979430 & 1.356736 & 1.946195 \\
$\mathrm{C}$ & -3.860691 & 1.192668 & 1.568051 \\
$\mathrm{H}$ & -3.645921 & 2.219824 & 1.852271 \\
$\mathrm{C}$ & -2.814240 & 0.368735 & 1.150819 \\
$\mathrm{H}$ & -1.794809 & 0.740164 & 1.118171 \\
$\mathrm{C}$ & 4.342513 & 1.611760 & 0.181010 \\
$\mathrm{H}$ & 4.541192 & 2.620724 & 0.553022 \\
$\mathrm{H}$ & 5.273797 & 1.175878 & -0.195591 \\
$\mathrm{C}$ & 3.619136 & 0.695355 & 1.220302 \\
$\mathrm{C}$ & 3.193322 & 1.470814 & 2.476408 \\
$\mathrm{H}$ & 2.601888 & 2.352777 & 2.207825 \\
$\mathrm{H}$ & 4.070787 & 1.801974 & 3.045135 \\
$\mathrm{H}$ & 2.580494 & 0.835939 & 3.123790 \\
$\mathrm{C}$ & 4.459162 & -0.532669 & 1.592983 \\
$\mathrm{H}$ & 3.891343 & -1.195616 & 2.253229 \\
$\mathrm{H}$ & 5.377282 & -0.232360 & 2.112312 \\
$\mathrm{H}$ & 4.736128 & -1.100363 & 0.698514 \\
$\mathrm{C}$ & 2.386776 & 0.883482 & -0.639181 \\
$\mathrm{C}$ & 1.313862 & 0.738530 & -1.615038 \\
$\mathrm{C}$ & 1.377748 & 1.419132 & -2.841166 \\
$\mathrm{H}$ & 2.239592 & 2.046353 & -3.042812 \\
$\mathrm{C}$ & 0.357500 & 1.291371 & -3.774713 \\
$\mathrm{H}$ & 0.412702 & 1.821226 & -4.720998 \\
$\mathrm{C}$ & -0.737280 & 0.474931 & -3.480498 \\
$\mathrm{H}$ & -1.543921 & 0.364697 & -4.200486 \\
$\mathrm{C}$ & -0.813017 & -0.206458 & -2.266956 \\
$\mathrm{H}$ & -1.668584 & -0.839292 & -2.059231 \\
$\mathrm{C}$ & 0.204935 & -0.087622 & -1.318389 \\
\hline & & & \\
\hline
\end{tabular}

\begin{tabular}{|lrrr|}
\hline \multicolumn{4}{c|}{ Compound 14 } \\
Se & 1.330539 & -1.692893 & 0.016070 \\
H & 2.804220 & -1.984580 & 0.028160 \\
O & -1.787677 & 1.620807 & -0.016900 \\
N & -1.187036 & -0.564578 & -0.010283 \\
C & 1.644903 & 0.197763 & -0.003831 \\
C & 2.954015 & 0.701338 & -0.006031 \\
H & 3.792670 & 0.012300 & 0.003792 \\
C & 3.200000 & 2.070804 & -0.020365 \\
H & 4.226922 & 2.426737 & -0.021399 \\
C & 2.139164 & 2.980093 & -0.033466 \\
H & 2.327639 & 4.049443 & -0.044951 \\
C & 0.836852 & 2.499258 & -0.031911 \\
H & -0.003060 & 3.184956 & -0.042259 \\
C & 0.572338 & 1.118915 & -0.017024 \\
C & -0.816641 & 0.658539 & -0.015388 \\
C & -3.048056 & 0.914454 & -0.052983 \\
H & -3.563824 & 1.188422 & -0.979454 \\
H & -3.646703 & 1.245353 & 0.801177 \\
C & -2.662527 & -0.598898 & 0.008317 \\
C & -3.133326 & -1.261631 & 1.311788 \\
H & -2.747617 & -2.283722 & 1.377485 \\
H & -4.228733 & -1.299819 & 1.355383 \\
H & -2.770929 & -0.706048 & 2.183422 \\
C & -3.173306 & -1.375660 & -1.212875 \\
H & -2.807888 & -2.406991 & -1.186307 \\
H & -2.820919 & -0.914953 & -2.141836 \\
H & -4.269928 & -1.396997 & -1.229816 \\
\hline
\end{tabular}




\begin{tabular}{|c|c|c|c|c|c|c|c|}
\hline \multicolumn{4}{|c|}{ Compound 15} & \multicolumn{4}{|c|}{ Compound 16} \\
\hline Se & -1.225059 & -1.256823 & 0.306194 & C & -0.811533 & 0.311072 & -0.059501 \\
\hline $\mathrm{O}$ & -3.072682 & -1.505912 & 0.488071 & C & $\begin{array}{l}-0.011503 \\
-2.170895\end{array}$ & 0.529004 & 0.177095 \\
\hline $\mathrm{O}$ & 2.277765 & 1.295663 & -0.433357 & $\mathrm{C}$ & -2.676928 & 1.829399 & 0.187818 \\
\hline $\mathrm{N}$ & 1.141044 & -0.606525 & 0.016369 & $\mathrm{C}$ & -1.831300 & 2.919378 & -0.026455 \\
\hline $\mathrm{C}$ & -1.318801 & 0.647906 & 0.060956 & C & $\begin{array}{l}-1.031300 \\
-0.473012\end{array}$ & 2.698863 & $\begin{array}{l}-0.0<0453 \\
-0.259353\end{array}$ \\
\hline $\mathrm{C}$ & -2.545132 & 1.319200 & 0.110015 & $\mathrm{C}$ & 0.044029 & 1.401949 & -0.288755 \\
\hline $\mathrm{H}$ & -3.445959 & 0.747939 & 0.303919 & $\mathrm{C}$ & 1.492272 & 1.120667 & -0.613935 \\
\hline $\mathrm{C}$ & -2.594669 & 2.698659 & -0.073146 & $\mathrm{~N}$ & 1.948945 & -0.051114 & 0.142180 \\
\hline $\mathrm{H}$ & -3.555883 & 3.204765 & -0.030290 & $\mathrm{C}$ & 3.081676 & -0.744666 & -0.464092 \\
\hline $\mathrm{C}$ & -1.428282 & 3.438931 & -0.306288 & $\mathrm{C}$ & 2.160799 & 0.226604 & 1.563191 \\
\hline $\mathrm{H}$ & -1.479549 & 4.514351 & -0.448297 & Se & -0.076258 & -1.476949 & -0.059806 \\
\hline $\mathrm{C}$ & -0.203655 & 2.785869 & -0.348484 & $\mathrm{O}$ & -1.733093 & -2.358901 & -0.158196 \\
\hline $\mathrm{H}$ & 0.717015 & 3.334369 & -0.521430 & $\mathrm{H}$ & -2.816510 & -0.325473 & 0.349623 \\
\hline $\mathrm{C}$ & -0.142367 & 1.396194 & -0.164096 & $\mathrm{H}$ & -3.735634 & 1.989708 & 0.375708 \\
\hline $\mathrm{C}$ & 1.104915 & 0.659961 & -0.187232 & $\begin{array}{l}H \\
H\end{array}$ & & & $\begin{array}{r}0.3 / 4 \\
0.00\end{array}$ \\
\hline $\mathrm{C}$ & 3.306871 & 0.277843 & -0.339396 & $\begin{array}{l}H \\
H\end{array}$ & -2.223255 & 3.932478 & -0.007709 \\
\hline $\mathrm{H}$ & 3.965864 & 0.542461 & 0.493824 & $\mathrm{H}$ & 0.194351 & 3.541830 & -0.427877 \\
\hline $\mathrm{H}$ & 3.879960 & 0.292947 & -1.270785 & $\mathrm{H}$ & 2.126141 & 2.005697 & -0.433932 \\
\hline C & 2.538359 & -1.064838 & -0.105339 & $\mathrm{H}$ & 1.584438 & 0.868188 & -1.679212 \\
\hline $\mathrm{C}$ & 2.654246 & -2.015800 & -1.306177 & $\mathrm{H}$ & 3.998889 & -0.132608 & -0.472412 \\
\hline $\mathrm{H}$ & 2.001078 & -2.882602 & -1.165266 & $\mathrm{H}$ & 2.829614 & -1.022776 & -1.491413 \\
\hline $\mathrm{H}$ & 3.684614 & -2.372812 & $\begin{array}{l}-1.105200 \\
-1.423241\end{array}$ & $\mathrm{H}$ & 3.284939 & -1.661258 & 0.098635 \\
\hline $\mathrm{H}$ & 2.354198 & -1512441 & $\begin{array}{l}-1.420<41 \\
-2231453\end{array}$ & $\mathrm{H}$ & 2.315998 & -0.716522 & 2.094899 \\
\hline II & & $\begin{array}{l}-1.51<441 \\
1761579\end{array}$ & -2.231453 & $\mathrm{H}$ & 3.033390 & 0.880180 & 1.732786 \\
\hline $\mathrm{C}$ & 2.970954 & -1.761529 & 1.191349 & $\mathrm{H}$ & 1.273033 & 0.711648 & 1.975710 \\
\hline $\mathrm{H}$ & 2.349031 & -2.643538 & 1.373500 & $\mathrm{H}$ & -1.941170 & -2.379430 & -1.107157 \\
\hline $\mathrm{H}$ & 2.863842 & -1.088526 & 2.048385 & & & & \\
\hline $\mathrm{H}$ & 4.017551 & -2.083612 & 1.130127 & & & & \\
\hline $\mathrm{H}$ & -3.382378 & -1.688031 & -0.414843 & & & & \\
\hline
\end{tabular}




\begin{tabular}{|lrrr|}
\hline \multicolumn{4}{c}{ Compound 17 } \\
Se & 0.596880 & 0.437045 & -0.909022 \\
S & 1.217928 & -1.741826 & -0.811630 \\
H & -2.278600 & 3.334025 & -1.725217 \\
N & -0.272373 & 2.849983 & -1.145143 \\
C & 1.659302 & -2.044168 & 0.910122 \\
C & 2.164651 & -3.316865 & 1.215533 \\
H & 2.276904 & -4.057790 & 0.428094 \\
C & 2.523007 & -3.631203 & 2.525589 \\
H & 2.913513 & -4.620834 & 2.749134 \\
C & 2.383045 & -2.685799 & 3.544325 \\
H & 2.662334 & -2.933494 & 4.564659 \\
C & 1.881896 & -1.420279 & 3.237404 \\
H & 1.768839 & -0.673785 & 4.019868 \\
C & 1.520658 & -1.095835 & 1.928944 \\
H & 1.140774 & -0.108775 & 1.684253 \\
H & -0.796198 & 4.454082 & 0.171284 \\
C & -0.354838 & 3.442088 & 0.185930 \\
H & -0.964261 & 2.807369 & 0.833877 \\
H & 0.650351 & 3.507056 & 0.613738 \\
C & -1.570912 & 2.486390 & -1.714450 \\
C & -2.156099 & 1.309079 & -0.968632 \\
C & -3.526858 & 1.226245 & -0.710033 \\
H & -4.174263 & 2.040490 & -1.028857 \\
C & -4.069818 & 0.118566 & -0.059448 \\
H & -5.138086 & 0.067555 & 0.131245 \\
C & -3.227016 & -0.913406 & 0.351922 \\
H & -3.632358 & -1.778883 & 0.869252 \\
C & -1.853764 & -0.839569 & 0.113703 \\
H & -1.207199 & -1.647428 & 0.437077 \\
$\mathrm{C}$ & -1.312841 & 0.262768 & -0.550012 \\
H & -1.391278 & 2.202149 & -2.760332 \\
C & 0.580869 & 3.611611 & -2.048442 \\
H & 0.182675 & 4.618320 & -2.262946 \\
H & 1.572923 & 3.723367 & -1.599239 \\
H & 0.692076 & 3.068634 & -2.991812 \\
\hline
\end{tabular}

\begin{tabular}{|lrrr|}
\hline \multicolumn{5}{c}{ Compound 18 } \\
Se & -3.171216 & -1.300179 & 2.463689 \\
S & -1.374510 & -0.026336 & 2.092506 \\
$\mathrm{O}$ & 0.858473 & 1.406331 & 1.670217 \\
$\mathrm{~N}$ & 1.615556 & 1.826912 & -0.456745 \\
$\mathrm{C}$ & -1.790125 & 0.938602 & 0.618405 \\
$\mathrm{C}$ & -0.772637 & 1.672278 & -0.040212 \\
$\mathrm{C}$ & -1.109644 & 2.469071 & -1.147222 \\
$\mathrm{H}$ & -0.344893 & 3.085405 & -1.612680 \\
$\mathrm{C}$ & -2.411418 & 2.529280 & -1.631618 \\
$\mathrm{H}$ & -2.650363 & 3.161399 & -2.481596 \\
$\mathrm{C}$ & -3.401712 & 1.776011 & -1.000356 \\
$\mathrm{H}$ & -4.426652 & 1.806267 & -1.360710 \\
$\mathrm{C}$ & -3.093096 & 0.992149 & 0.107794 \\
$\mathrm{H}$ & -3.869456 & 0.414287 & 0.597963 \\
$\mathrm{C}$ & 0.629400 & 1.625907 & 0.482231 \\
$\mathrm{C}$ & 3.015815 & 1.869959 & -0.280893 \\
$\mathrm{C}$ & 3.642703 & 1.808000 & 0.973258 \\
$\mathrm{H}$ & 3.042595 & 1.703872 & 1.865751 \\
$\mathrm{C}$ & 5.034599 & 1.871848 & 1.043269 \\
$\mathrm{H}$ & 5.511105 & 1.822195 & 2.018799 \\
$\mathrm{C}$ & 5.814955 & 1.994933 & -0.106773 \\
$\mathrm{H}$ & 6.897729 & 2.042257 & -0.035238 \\
$\mathrm{C}$ & 5.186366 & 2.055213 & -1.351972 \\
$\mathrm{H}$ & 5.774913 & 2.150435 & -2.260482 \\
$\mathrm{C}$ & 3.798937 & 1.994166 & -1.439995 \\
$\mathrm{H}$ & 3.315346 & 2.042985 & -2.414186 \\
$\mathrm{C}$ & -2.767626 & -2.828161 & 1.358298 \\
$\mathrm{C}$ & -1.947420 & -3.841481 & 1.865957 \\
$\mathrm{H}$ & -1.523077 & -3.735685 & 2.859431 \\
$\mathrm{C}$ & -1.674578 & -4.969747 & 1.092676 \\
$\mathrm{H}$ & -1.033160 & -5.752737 & 1.488111 \\
$\mathrm{C}$ & -2.227966 & -5.092635 & -0.184003 \\
$\mathrm{H}$ & -2.017447 & -5.973291 & -0.784876 \\
$\mathrm{C}$ & -3.055127 & -4.086262 & -0.686713 \\
$\mathrm{H}$ & -3.488740 & -4.181180 & -1.678743 \\
$\mathrm{C}$ & -3.327423 & -2.953691 & 0.082550 \\
$\mathrm{H}$ & -3.969615 & -2.170177 & -0.306642 \\
$\mathrm{H}$ & 1.309405 & 1.851801 & -1.419252 \\
& & & \\
\hline & & & \\
\hline
\end{tabular}




\begin{tabular}{|lccl|}
\hline & \multicolumn{3}{c}{ Compound 19 } \\
S & .284190 & .238090 & .961169 \\
Se & -.307378 & 2.378785 & 1.234451 \\
$\mathrm{O}$ & -.019011 & -4.051493 & -.287903 \\
$\mathrm{~N}$ & 1.154573 & -2.352340 & .646693 \\
$\mathrm{C}$ & .127707 & 3.096420 & -.502233 \\
$\mathrm{C}$ & 1.431655 & 3.542630 & -.742973 \\
$\mathrm{H}$ & 2.179773 & 3.443241 & .037255 \\
$\mathrm{C}$ & 1.761316 & 4.100602 & -1.978268 \\
$\mathrm{H}$ & 2.776208 & 4.442778 & -2.162507 \\
$\mathrm{C}$ & .788240 & 4.219574 & -2.973019 \\
$\mathrm{H}$ & 1.044752 & 4.655498 & -3.934863 \\
$\mathrm{C}$ & -.515421 & 3.781781 & -2.730387 \\
$\mathrm{H}$ & -1.274665 & 3.875041 & -3.502375 \\
$\mathrm{C}$ & -.848874 & 3.222393 & -1.495824 \\
$\mathrm{H}$ & -1.861597 & 2.881241 & -1.307564 \\
$\mathrm{C}$ & 1.266183 & -4.639412 & .011013 \\
$\mathrm{H}$ & 1.655763 & -5.087560 & -.907742 \\
$\mathrm{H}$ & 1.114928 & -5.426625 & .758136 \\
$\mathrm{C}$ & 2.132108 & -3.455065 & .544482 \\
$\mathrm{C}$ & 3.233205 & -3.053432 & -.449297 \\
$\mathrm{H}$ & 2.808588 & -2.854261 & -1.439204 \\
$\mathrm{H}$ & 3.979997 & -3.851237 & -.545780 \\
$\mathrm{H}$ & 3.737697 & -2.144405 & -.107795 \\
$\mathrm{C}$ & 2.726062 & -3.749248 & 1.927867 \\
$\mathrm{H}$ & 3.256367 & -2.870603 & 2.307922 \\
$\mathrm{H}$ & 3.433660 & -4.586076 & 1.878329 \\
$\mathrm{H}$ & 1.936978 & -4.004820 & 2.643019 \\
$\mathrm{C}$ & .044644 & -2.759577 & .162741 \\
$\mathrm{C}$ & -1.181741 & -1.965662 & .043264 \\
$\mathrm{C}$ & -2.349638 & -2.600254 & -.414101 \\
$\mathrm{H}$ & -2.292873 & -3.651853 & -.671393 \\
$\mathrm{C}$ & -3.548165 & -1.909109 & -.532912 \\
$\mathrm{H}$ & -4.439990 & -2.417691 & -.886904 \\
$\mathrm{C}$ & -3.587290 & -.558085 & -.185556 \\
$\mathrm{H}$ & -4.516078 & .001713 & -.261029 \\
$\mathrm{C}$ & -2.439670 & .089937 & .263020 \\
$\mathrm{H}$ & -2.480845 & 1.138113 & .538265 \\
$\mathrm{C}$ & -1.221116 & -.588878 & .383945 \\
\hline & & & \\
\hline
\end{tabular}

\begin{tabular}{|c|c|c|c|}
\hline \multicolumn{4}{|c|}{ Compound 20} \\
\hline Se & 0.166797 & -0.998253 & 0.167767 \\
\hline Se & -2.114002 & -1.584346 & 0.319534 \\
\hline $\mathrm{O}$ & 3.696606 & 1.203527 & -1.497182 \\
\hline $\mathrm{N}$ & 2.678443 & -0.271623 & -0.116052 \\
\hline $\mathrm{C}$ & -2.864251 & -0.125328 & 1.353360 \\
\hline $\mathrm{C}$ & -4.246293 & -0.149433 & 1.574533 \\
\hline $\mathrm{H}$ & -4.849744 & -0.957427 & 1.167976 \\
\hline $\mathrm{C}$ & -4.852089 & 0.864378 & 2.316993 \\
\hline $\mathrm{H}$ & -5.925603 & 0.835331 & 2.485483 \\
\hline $\mathrm{C}$ & -4.086989 & 1.910686 & 2.836169 \\
\hline $\mathrm{H}$ & -4.560094 & 2.701333 & 3.411967 \\
\hline $\mathrm{C}$ & -2.710472 & 1.933731 & 2.606108 \\
\hline $\mathrm{H}$ & -2.104178 & 2.744514 & 3.002340 \\
\hline $\mathrm{C}$ & -2.097026 & 0.921152 & 1.867171 \\
\hline $\mathrm{H}$ & -1.026522 & 0.937876 & 1.685651 \\
\hline $\mathrm{C}$ & 4.774943 & 0.680338 & -0.684409 \\
\hline $\mathrm{H}$ & 5.289098 & 1.527250 & -0.221295 \\
\hline $\mathrm{H}$ & 5.470987 & 0.153727 & -1.345809 \\
\hline $\mathrm{C}$ & 4.080938 & -0.267196 & 0.346614 \\
\hline $\mathrm{C}$ & 4.134274 & 0.295283 & 1.775372 \\
\hline $\mathrm{H}$ & 3.738191 & 1.316028 & 1.807171 \\
\hline $\mathrm{H}$ & 5.165407 & 0.311868 & 2.148985 \\
\hline $\mathrm{H}$ & 3.531965 & -0.322981 & 2.448193 \\
\hline $\mathrm{C}$ & 4.648886 & -1.691238 & 0.297628 \\
\hline $\mathrm{H}$ & 4.085287 & -2.348639 & 0.967015 \\
\hline $\mathrm{H}$ & 5.700462 & -1.701163 & 0.609074 \\
\hline $\mathrm{H}$ & 4.583186 & -2.101549 & -0.715510 \\
\hline $\mathrm{C}$ & 2.572113 & 0.553576 & -1.087995 \\
\hline $\mathrm{C}$ & 1.319351 & 0.844801 & -1.776178 \\
\hline $\mathrm{C}$ & 1.301835 & 1.737683 & -2.859753 \\
\hline $\mathrm{H}$ & 2.234748 & 2.195551 & -3.170840 \\
\hline $\mathrm{C}$ & 0.114021 & 2.028316 & -3.518397 \\
\hline $\mathrm{H}$ & 0.108500 & 2.720177 & -4.355448 \\
\hline $\mathrm{C}$ & -1.069639 & 1.422319 & -3.089823 \\
\hline $\mathrm{H}$ & -2.007990 & 1.639792 & -3.593249 \\
\hline $\mathrm{C}$ & -1.066784 & 0.535487 & -2.015294 \\
\hline $\mathrm{H}$ & -1.990535 & 0.068367 & -1.689330 \\
\hline $\mathrm{C}$ & 0.120489 & 0.232355 & -1.345567 \\
\hline
\end{tabular}




\begin{tabular}{|lrrr|}
\hline & & & \\
& & & \\
$\mathrm{S}$ & -0.421713 & 0.608236 & -0.751654 \\
$\mathrm{Se}$ & 1.417873 & -0.378392 & -1.569923 \\
$\mathrm{H}$ & -4.493405 & 0.842548 & 0.070953 \\
$\mathrm{~N}$ & -2.681084 & 1.943224 & 0.361156 \\
$\mathrm{C}$ & 2.666096 & -0.022929 & -0.142604 \\
$\mathrm{C}$ & 3.381450 & 1.179516 & -0.160822 \\
$\mathrm{H}$ & 3.190926 & 1.897752 & -0.952121 \\
$\mathrm{C}$ & 4.322234 & 1.448791 & 0.833629 \\
$\mathrm{H}$ & 4.872067 & 2.386082 & 0.817179 \\
$\mathrm{C}$ & 4.555585 & 0.514871 & 1.845213 \\
$\mathrm{H}$ & 5.289061 & 0.723685 & 2.619433 \\
$\mathrm{C}$ & 3.848826 & -0.689613 & 1.859475 \\
$\mathrm{H}$ & 4.030657 & -1.419195 & 2.644218 \\
$\mathrm{C}$ & 2.906527 & -0.961583 & 0.866407 \\
$\mathrm{H}$ & 2.357250 & -1.897578 & 0.879347 \\
$\mathrm{H}$ & -3.805913 & 2.192211 & 2.168153 \\
$\mathrm{C}$ & -2.778653 & 1.977038 & 1.814085 \\
$\mathrm{H}$ & -2.468661 & 1.013884 & 2.228626 \\
$\mathrm{H}$ & -2.111227 & 2.750713 & 2.206995 \\
$\mathrm{C}$ & -3.428748 & 0.838285 & -0.233701 \\
$\mathrm{C}$ & -2.816152 & -0.508137 & 0.084072 \\
$\mathrm{C}$ & -3.616039 & -1.543120 & 0.579787 \\
$\mathrm{H}$ & -4.667039 & -1.339973 & 0.773476 \\
$\mathrm{C}$ & -3.101952 & -2.817639 & 0.815398 \\
$\mathrm{H}$ & -3.745330 & -3.604802 & 1.198314 \\
$\mathrm{C}$ & -1.757531 & -3.066731 & 0.545853 \\
$\mathrm{H}$ & -1.337354 & -4.055791 & 0.709562 \\
$\mathrm{C}$ & -0.937682 & -2.047437 & 0.063031 \\
$\mathrm{H}$ & 0.098754 & -2.250675 & -0.183884 \\
$\mathrm{C}$ & -1.447423 & -0.764549 & -0.157287 \\
$\mathrm{H}$ & -3.409336 & 0.988578 & -1.321821 \\
$\mathrm{C}$ & -3.005447 & 3.227456 & -0.240981 \\
$\mathrm{H}$ & -4.057457 & 3.531135 & -0.076216 \\
$\mathrm{H}$ & -2.359127 & 4.002504 & 0.183911 \\
$\mathrm{H}$ & -2.823095 & 3.184603 & -1.319685 \\
\hline & & &
\end{tabular}

\begin{tabular}{|lccc|}
\hline & \multicolumn{3}{c}{ Compound 26 } \\
S & 1.213611 & -0.881359 & -1.436601 \\
Se & -0.589783 & -1.396676 & -0.208201 \\
O & -2.724745 & -1.904099 & 1.128495 \\
H & -4.299065 & -0.723184 & 1.607350 \\
C & -1.554070 & 0.280748 & -0.250446 \\
C & -2.802715 & 0.343567 & 0.413929 \\
C & -3.543557 & 1.537794 & 0.421543 \\
C & -4.500040 & 1.558356 & 0.939309 \\
$\mathrm{C}$ & -3.064339 & 2.670690 & -0.219585 \\
C & -3.636223 & 3.593455 & -0.213262 \\
C & -1.829211 & 2.604706 & -0.874988 \\
H & -1.439090 & 3.483438 & -1.381815 \\
C & -1.081625 & 1.427858 & -0.891801 \\
H & -0.130001 & 1.401659 & -1.411028 \\
C & -3.320958 & -0.832037 & 1.099497 \\
C & 2.388859 & -0.138508 & -0.283531 \\
C & 2.120475 & 0.090622 & 1.068459 \\
H & 1.158174 & -0.196849 & 1.480032 \\
C & 3.095841 & 0.673898 & 1.878885 \\
H & 2.877930 & 0.846486 & 2.929758 \\
C & 4.337270 & 1.032223 & 1.352595 \\
H & 5.092074 & 1.486384 & 1.988336 \\
C & 4.601791 & 0.800376 & 0.001035 \\
H & 5.565371 & 1.072431 & -0.421926 \\
C & 3.634931 & 0.218049 & -0.817542 \\
H & 3.847505 & 0.039451 & -1.868558 \\
\hline & & & \\
\hline
\end{tabular}




\begin{tabular}{|lrcc|}
\hline & Compound 27 & \\
S & 0.616367 & 0.527815 & 0.930972 \\
Se & -1.568485 & 0.175090 & 1.344281 \\
$\mathrm{O}$ & 4.257831 & 1.975007 & -1.289096 \\
$\mathrm{~N}$ & 3.326769 & 1.059880 & 0.566415 \\
$\mathrm{C}$ & -1.788786 & -1.630976 & 0.692644 \\
$\mathrm{O}$ & -0.762739 & -2.352645 & 0.076712 \\
$\mathrm{H}$ & 0.215857 & -1.900931 & -0.042146 \\
$\mathrm{C}$ & -0.983154 & -3.652610 & -0.376032 \\
$\mathrm{H}$ & -0.166705 & -4.190720 & -0.850653 \\
$\mathrm{C}$ & -2.231045 & -4.268821 & -0.229406 \\
$\mathrm{H}$ & -2.391752 & -5.281657 & -0.585914 \\
$\mathrm{C}$ & -3.258233 & -3.561821 & 0.378522 \\
$\mathrm{H}$ & -4.239458 & -4.014103 & 0.506181 \\
$\mathrm{C}$ & -3.053796 & -2.250566 & 0.840996 \\
$\mathrm{C}$ & 5.382216 & 1.801769 & -0.395752 \\
$\mathrm{H}$ & 5.857686 & 2.776567 & -0.255314 \\
$\mathrm{H}$ & 6.094718 & 1.122231 & -0.873866 \\
$\mathrm{C}$ & 4.746142 & 1.222470 & 0.895226 \\
$\mathrm{H}$ & 4.851595 & 1.894447 & 1.755285 \\
$\mathrm{H}$ & 5.178618 & 0.256908 & 1.181153 \\
$\mathrm{C}$ & 3.158537 & 1.498126 & -0.622076 \\
$\mathrm{C}$ & 1.888364 & 1.543933 & -1.355525 \\
$\mathrm{C}$ & 1.904559 & 2.012542 & -2.681060 \\
$\mathrm{H}$ & 2.853918 & 2.321972 & -3.102619 \\
$\mathrm{C}$ & 0.743086 & 2.081526 & -3.439151 \\
$\mathrm{H}$ & 0.778899 & 2.446225 & -4.461427 \\
$\mathrm{C}$ & -0.463571 & 1.678017 & -2.867421 \\
$\mathrm{H}$ & -1.386582 & 1.725232 & -3.439398 \\
$\mathrm{C}$ & -0.502341 & 1.211249 & -1.556304 \\
$\mathrm{H}$ & -1.449034 & 0.915040 & -1.118173 \\
$\mathrm{C}$ & 0.658973 & 1.133252 & -0.776782 \\
$\mathrm{C}$ & -4.163222 & -1.538062 & 1.466288 \\
$\mathrm{H}$ & -5.121333 & -2.091505 & 1.537336 \\
$\mathrm{O}$ & -4.076223 & -0.395164 & 1.898878 \\
\hline & & &
\end{tabular}


(S1) Frisch, M. J.; Trucks, G. W.; Schlegel, H. B.; Scuseria, G. E.; Robb, M. A.;

Cheeseman, J. R.; Zakrzewski, V. G.; Montgomery, J. A.; Stratmann, R. E.; Burant, J. C.; Dapprich, S.; Millam, J. M.; Daniels, A. D.; Kudin, K. N.; Strain, M. C.;

Farkas, O.; Tomasi, J.; Barone, V.; Cossi, M.; Cammi, R.; Mennucci, B.; Pomelli, C.; Adamo, C.; Clifford, S.; Ochterski, J.; Petersson, G. A.; Ayala, P. Y.; Cui, Q.;

Morokuma, K.; Malick, D. K.; Rabuck, A. D.; Raghavachari, K.; Foresman, J. B.; Cioslowski, J.; Ortiz, J. V.; Baboul, A. G.; Stefanov, B. B.; Liu, G.; Liashenko, A.; Piskorz, P.; Komaromi, I.; Gomperts, R.; Martin, R. L.; Fox, D. J.; Keith, T.; AlLaham, M. A.; Peng, C. Y.; Nanayakkara, A.; Gonzalez, C.; Challacombe, M.; Gill, P. M. W.; Johnson, B.; Chen, W.; Wong, M. W.; Andres, J. L.; Gonzalez, C.; HeadGordon, M.; Replogle, E. S.; Pople, J. A. Gaussian 98; Gaussian, Inc.: Pittsburgh, PA, 1998.

(S2) Mugesh, G.; du Mont, W.-W.; Wismach, C.; Jones, P. G. ChemBioChem. 2002, 3, 440. 